\title{
Slope-Assisted Brillouin-Based Distributed Fiber-Optic Sensing Techniques
}

\author{
Xinyu Fan $\mathbb{D}^{1},{ }^{1}$ Bin Wang, ${ }^{1,2,3,4}$ Guangyao Yang, ${ }^{1}$ and Zuyuan $H e \mathbb{D}^{1}$ \\ ${ }^{1}$ State Key Laboratory of Advanced Optical Communication Systems and Networks, Department of Electronic Engineering, \\ Shanghai Jiao Tong University, Shanghai 200240, China \\ ${ }^{2}$ Radar Research Lab, School of Information and Electronics, Beijing Institute of Technology, Beijing 100081, China \\ ${ }^{3}$ Key Laboratory of Electronic and Information Technology in Satellite Navigation (Beijing Institute of Technology), \\ Ministry of Education, Beijing 100081, China \\ ${ }^{4}$ Beijing Institute of Technology Chongqing Innovation Center, Chongqing 401120, China
}

Correspondence should be addressed to Xinyu Fan; fan.xinyu@sjtu.edu.cn and Zuyuan He; zuyuanhe@sjtu.edu.cn

Received 18 January 2021; Accepted 27 May 2021; Published 14 July 2021

Copyright ( 2021 Xinyu Fan et al. Exclusive Licensee Beijing Institute of Aerospace Control Devices. Distributed under a Creative Commons Attribution License (CC BY 4.0).

\begin{abstract}
Brillouin-based fiber-optic sensing has been regarded as a powerful distributed measurement tool for monitoring the conditions of modern large civil and geotechnical structures, since it provides continuous environmental information (e.g., temperature and strain) along the whole fiber used for sensing applications. In the past few decades, great research efforts were devoted to improve its performance in terms of measurement range, spatial resolution, measurement speed, sensitivity, and costeffectiveness, of which the slope-assisted measurement scheme, achieved by exploiting the linear slope of the Brillouin gain spectrum (BGS), have paved the way for dynamic distributed fiber-optic sensing. In this article, slope-assisted Brillouin-based distributed fiber-optic sensing techniques demonstrated in the past few years will be reviewed, including the slope-assisted Brillouin optical time-domain analysis/reflectometry (SA-BOTDA/SA-BOTDR), the slope-assisted Brillouin dynamic grating (BDG) sensor, and the slope-assisted Brillouin optical correlation domain analysis/reflectometry (SA-BOCDA/SA-BOCDR). Avenues for future research and development of slope-assisted Brillouin-based fiber-optic sensors are also prospected.
\end{abstract}

\section{Introduction}

Recently, aging degradation and seismic damage of civil infrastructures such as bridges, pipelines, and buildings have posed a serious issue for public security. Diagnosing the health of structures in a fully distributed manner could help prevent the structural collapse of buildings and other civil works. Distributed fiber-optic sensing is a promising technology due to its unique advantages, including a large number of effective sensing points, high sensitivity, long measurement range, high spatial resolution, immunity to electromagnetic interference, small size, and light weight.

Brillouin-based distributed fiber-optic sensing, invented in the late 1980's, is one of the most important fiber-optic sensing techniques since it reveals the temperature and strain information by simply measuring the Brillouin frequency shift (BFS) distribution along with the fiber under test (FUT) [1]. Generally, the Brillouin-based fiber-optic sensors can be classified into two categories: "analysis" based on stimulated Brillouin scattering (SBS) and "reflectometry" based on spontaneous Brillouin scattering (SpBS). For each category, various distributed sensing techniques have been proposed, including Brillouin optical time-domain analysis/reflectometry (BOTDA/R) [1-4], Brillouin optical frequency-domain analysis/reflectometry (BOFDA/R) [5-8], and Brillouin optical correlation domain analysis/reflectometry (BOCDA/R) [9-13]. Furthermore, great research efforts have been devoted to further extend the application fields of these techniques through recently achieved enhanced performance in each of its critical dimensions: measurement range has been extended to hundreds of kilometers $[14,15]$; spatial resolution is of the order of a few millimeters [16-19]; dynamic events can be captured at sampling rates as 
high as $\mathrm{MHz}[20,21]$; temperature/strain sensitivity has been enhanced by a factor of six by exploiting the high-order Stokes waves [22, 23].

Generally, in a Brillouin-based sensing system, the whole Brillouin gain spectrum (BGS) is supposed to be measured by sweeping the frequency interval between the pump wave and Stoke wave, to extract the BFS and therefore to obtain the temperature/strain information along the FUT. Nevertheless, the frequency sweeping process used to obtain the BGS is relatively time-consuming and therefore limits the sampling rate of the Brillouin-based sensing system. Recently, a novel measurement scheme called slope-assisted technique has been proposed by exploiting the linear slope of the BGS [24]. In this scheme, the frequency interval between the pump wave and probe (or reference) wave is fixed at the middle of the BGS linear slope, to convert the temperature/ strain-induced BFS change to Brillouin signal power variation, where the frequency sweeping process is avoided and the sampling rate is improved significantly.

In this article, the theoretical principles of the slopeassisted Brillouin-based sensing technique are introduced firstly, which is presented in Section 2. Then, in Section 3, the recent research progress on slope-assisted Brillouin timedomain sensing systems is reviewed, including slope-assisted BOTDA (SA-BOTDA), slope-assisted BOTDR (SA-BOTDR), and slope-assisted Brillouin dynamic grating (BDG) sensor. In Section 4, recent advancement on slope-assisted Brillouin correlation domain sensing systems is reviewed, including slopeassisted BOCDA (SA-BOCDA) and slope-assisted BOCDR (SA-BOCDR). At last, the possible future research directions in this area are discussed in Section 5.

\section{Theoretical Principles}

Brillouin-based fiber-optic sensing is implemented by using the Brillouin nonlinear process in the optical fiber, where acoustic phonons scatter a forward propagating optical pump wave into a backward propagating Brillouin signal wave. The backscattered lightwave has a characteristic frequency shift from that of the pump wave, called BFS, which changes linearly with the local temperature and the strain applied to the optical fiber. For standard single-mode fibers (SMFs) pumped at wavelengths around $1550 \mathrm{~nm}$, the temperature and strain coefficients are about $1 \mathrm{MHz} /{ }^{\circ} \mathrm{C}$ and $0.05 \mathrm{MHz} / \mu \varepsilon$, respectively [25]. Therefore, using appropriate interrogation techniques, the distributed temperature/strain information can be extracted from the BFS distribution along the whole FUT.

In a Brillouin-based sensing system, to obtain the temperature/strain information, the BGS is measured either by using a Brillouin amplification scheme where SBS process is introduced, or by using a Brillouin generation scheme where SpBS is generated [26]. Mathematically, when a pump wave is launched into the optical fiber, the Brillouin gain of the probe (or Stokes) wave can be given by

$$
\begin{aligned}
G_{S}\left(z, v, v_{B}(z), P(z)\right) & =K \cdot P(z) \cdot B G S\left(\left[v-v_{B}(z)\right] / \Delta v_{B}\right) \\
& =K \cdot P(z) \cdot \frac{g_{0}}{1+4\left(v-v_{B}(z)\right)^{2} /\left(\Delta v_{B}\right)^{2}},
\end{aligned}
$$

where $P(z)$ is the pump power at $z, g_{0}$ is the Brillouin gain coefficient, $v$ is the frequency interval between the pump wave and probe wave (or Stokes), $v_{B}(z)$ is the BFS at location $z$, whose typical value is $11 \mathrm{GHz}$ for $\mathrm{SMF}, \Delta v_{B}$ is the full width as half maximum (FWHM) of the BGS, whose typical value is $35 \mathrm{MHz}$, and $K$ is a coefficient determined by material constants and the relative polarizations of the pump wave and Stoke wave.

Figure 1(a) shows the schematic illustration of the slopeassisted measurement scheme. When the ambient temperature is increased or a strain is applied to the optical fiber, the measured BGS curve will shift to a higher frequency, and the Brillouin signal power at a certain pump-probe (or reference) frequency interval will change accordingly. When the frequency interval is set at the linear slope of the BGS, the strain/temperature-induced BFS change is linearly converted to the Brillouin signal power variation. In this case, the timeconsuming frequency sweeping process is avoided, and the measurement speed increases drastically. Figure 1(b) shows that when a dynamic strain is applied to a fiber, the Brillouin signal power at a certain pump-probe (or reference) frequency interval (within the linear-slope range) will change at the same frequency of the dynamic strain. This means that dynamic strains can be measured with a high sampling rate by using the slope-assisted measurement scheme.

\section{Slope-Assisted Brillouin Time-Domain Sensors}

Investigators have proposed a variety of slope-assisted Brillouin time-domain sensing systems, such as SA-BOTDA [24, 29-41], SA-BOTDR [42], and slope-assisted Brillouin dynamic grating (BDG) sensor [43-45].

3.1. SA-BOTDA. Up to now, various techniques have been proposed to improve the performance of SA-BOTDA in each of its critical dimensions: SA-BOTDA with specially synthesized probe wave has been proposed for distributed dynamic sensing in optical fibers with arbitrary Brillouin profile [24, 29]; pump-power-independent measurements can be implemented by double-SA-BOTDA [31]; and a much wider strain dynamic range can be realized by a variety of techniques, such as SA-BOTDA based on Brillouin Phase-Gain Ratio [32], multi-SA-BOTDA [33-35], and SA-BOTDA enhanced by BGS engineering $[40,41]$.

3.1.1. SA-BOTDA-Based Sensing in Optical Fibers with Arbitrary Brillouin Profile. The slope-assisted concept is firstly introduced into BOTDA by Peled et al. in 2011 [24], to implement distributed measurement of fast strain variations along the entire fiber used for sensing applications. In this scheme, instead of using a CW probe wave and a swept frequency pump pulse, a pump pulse with a fixed optical frequency and a CW probe wave with variable optical frequency are employed. Since the BFS has a nonuniform spatial distribution along the FUT, the static BGS is firstly measured by using classical BOTDA, and the BFS distribution is extracted accurately. To implement distributed dynamic strain measurement, the frequency of the probe wave is temporally 


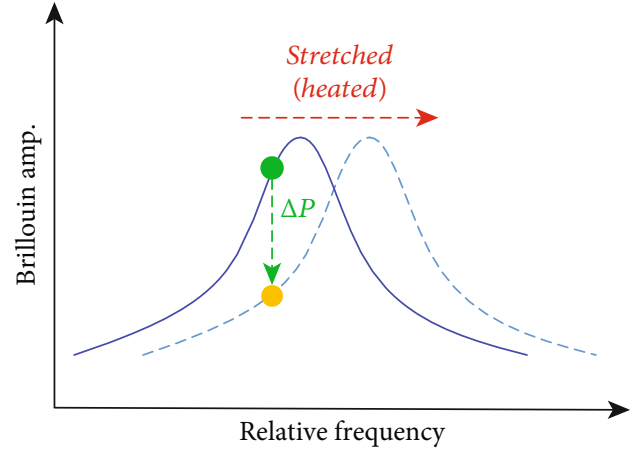

(a)

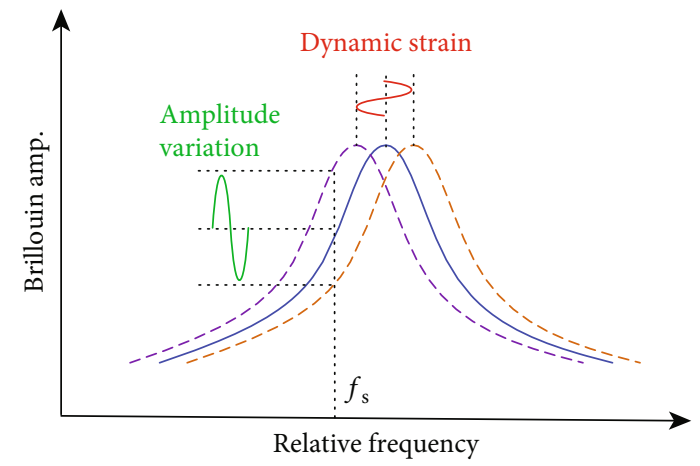

(b)

FIGURE 1: The schematic illustration of slope-assisted measurement scheme. (a) Strain/temperature variation induces a frequency shift as well as Brillouin power change. (b) Dynamic strain leads to Brillouin power variation when the pump-probe frequency interval is fixed [27, 28].

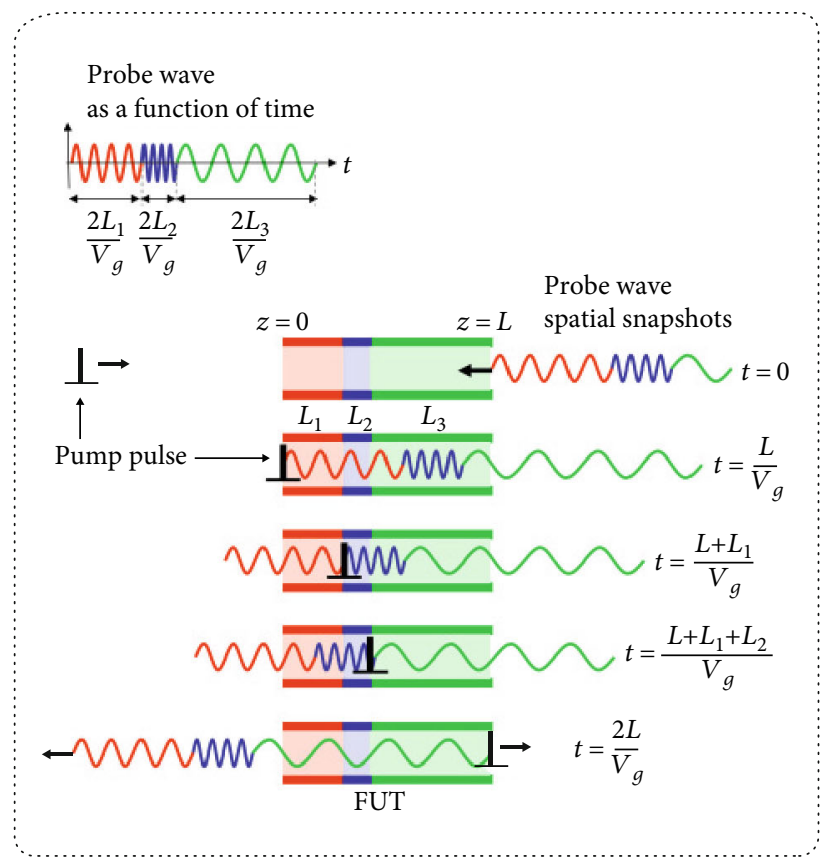

(a)

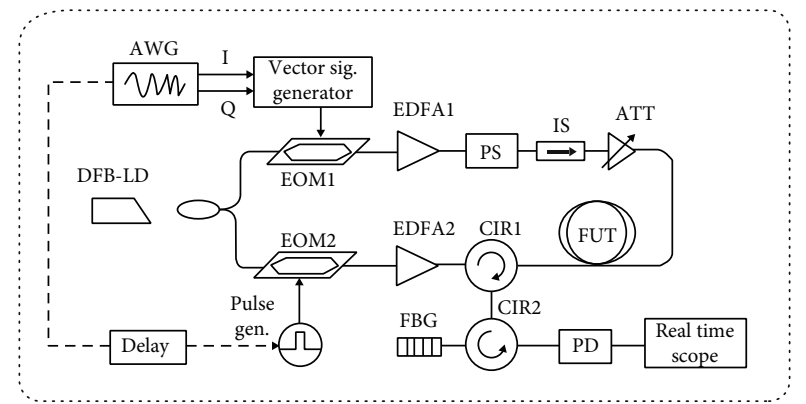

(b)

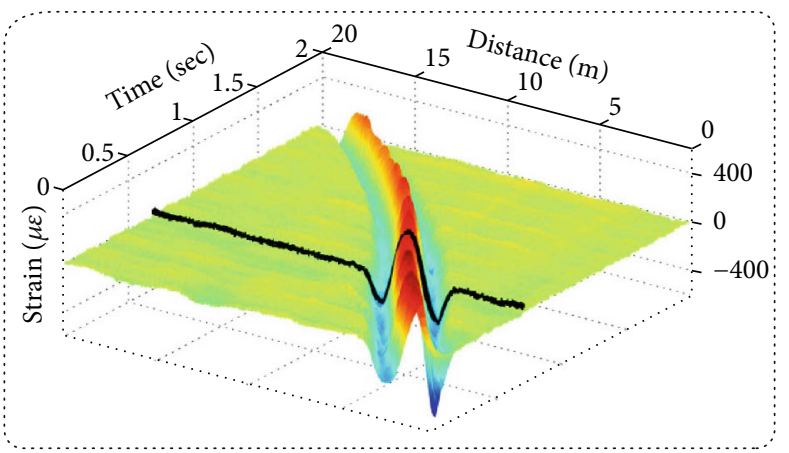

(c)

FIGURE 2: (a) Schematic illustration of the proposed measurement scheme, where a pump pulse interacts with a counter-propagating complex probe wave, which comprises 3 different optical frequencies [24]. (b) Experimental setup of the SA-BOTDA. AWG: arbitrary waveform generator; EOM: electro-optic modulator; EDFA: erbium-doped fiber amplifier; CIR: circulator; FBG: fiber Bragg grating; PS: polarization scrambler; IS: isolator; ATT: attenuator; FUT: fiber under test; PD: photodiode. (c) 3D view of the strain distribution when a mechanical wave is propagated along the FUT [29].

tailored in such a way that when the probe wave meets the counter-propagating pump pulse at an arbitrary location along the fiber, the optical frequency difference between these two waves sits as close as possible to the middle of the BGS slope, as shown in Figure 2(a). In this way, the straininduced BFS change is linearly converted to the Brillouin power change, and the time-consuming frequency sweeping process is avoided. Therefore, the measurement speed of the sensing system is greatly improved, and the sampling rate is limited only by the fiber length $L$ and the averaging number $N_{a} v g$ to a value bounded by $1 /\left[2 N_{a} v g L / v_{g}\right]$, where $v_{g}$ is the fiber group velocity.
Figure 2(b) shows the detailed experimental setup, which is similar to the conventional BOTDA system, except for an arbitrary waveform generator (AWG) employed to generate complex probe wave. By using the proposed technique, strain vibrations of a few hundred $\mathrm{Hz}$ were measured with a high measurement accuracy of $0.25 \mathrm{MHz}(5 \mu \varepsilon)$. The measurement range and spatial resolution are $85 \mathrm{~m}$ and $1.5 \mathrm{~m}$, respectively, and the strain dynamic range is $30 \mathrm{MHz}(600 \mu \varepsilon)$, which is determined by the linear slope range of the BGS. Subsequently, the SA-BOTDA is used for monitoring the propagation of the mechanical wave, and the $3 \mathrm{D}$ view of the strain distribution is shown in Figure 2(c). The 
propagation velocity of the mechanical wave was measured to be $9 \mathrm{~m} / \mathrm{s}$, which matches well with the results measured by the FBG sensors.

3.1.2. Double-SA-BOTDA. A practical problem with SABOTDA is the dependence of the conversion factor on variations in the optical power of the pump wave. To solve this problem, a double-SA-BOTDA technique was proposed by Motil et al. in 2014 [31], where the temperature/strain information is extracted from the ratio between readings taken on both slopes of the BGS. In this method, the pump-probe frequency interval $v$ is set at either the positive slope $\left(v^{+}\right)$or the negative slope $\left(v^{-}\right)$of the BGS, and the Brillouin gain for these two cases can be given by [31]

$$
G_{S-\text { Pos. }}\left(z, v^{+}, v_{B}(z), P(z)\right)=K \cdot P(z) \cdot B G S\left(\frac{\left[v^{+}-v_{B}(z)\right]}{\Delta v_{B}}\right),
$$

$$
G_{S-\mathrm{Neg} .}\left(z, v^{-}, v_{B}(z), P(z)\right)=K \cdot P(z) \cdot B G S\left(\frac{\left[v^{-}-v_{B}(z)\right]}{\Delta v_{B}}\right) .
$$

According to Equations (2) and (3), the Brillouin gain is sensitive to not only the BFS $v_{B}(z)$ but also the pump power $P(z)$, which may lead to a deteriorated measurement accuracy. When double-SA-BOTDA is implemented, the ratio between readings taken on both slopes of the BGS can be expressed as [31]

$$
\begin{aligned}
R_{B}\left(z, v^{+}, v^{-}, v_{B}(z)\right) & =\frac{G_{S-\text { Pos. }}\left(z, v^{+}, v_{B}(z), P(z)\right)}{G_{S-N e g .}\left(z, v^{-}, v_{B}(z), P(z)\right)} \\
& =\frac{B G S\left(\left[v^{+}-v_{B}(z)\right] / \Delta v_{B}\right)}{B G S\left(\left[v^{-}-v_{B}(z)\right] / \Delta v_{B}\right)} .
\end{aligned}
$$

It can be observed that the ratio is a function of the BFS $v_{B}(z)$ but is independent on the pump power $P(z)$. However, since the pump-probe frequency interval is sequentially changed between $v^{+}$and $v^{-}$, the ultimate sampling rate is halved compared with the conventional SA-BOTDA.

The experimental setup of the double-SA-BOTDA is similar to SA-BOTDA. Figure 3(a) shows the measured Brillouin gain change using the conventional SA-BOTDA when $55-\mathrm{Hz}$ strain vibrations are applied to the FUT in the presence of $>6 \mathrm{~dB}$ pump power variation, and Figure 3(b) shows the extracted $55 \mathrm{~Hz}$ strain variations using double-SA-BOTDA, where the effect induced by the pump power variation is eliminated, which matches well with the theoretical analysis. By using the proposed method, a sampling rate of $1 \mathrm{kHz}$ and a spatial resolution of $1.5 \mathrm{~m}$ over a 13-m-long FUT were realized with good immunity to pump power variation.

3.1.3. SA-BOTDA Based on Brillouin Phase-Gain Ratio. In the abovementioned SA-BOTDA systems, the strain dynamic range is normally limited to $30 \mathrm{MHz}(600 \mu \varepsilon)$, which is determined by the linear slope range of the BGS. To extend the strain dynamic range, a novel technique by introducing a new parameter called Brillouin phase-gain

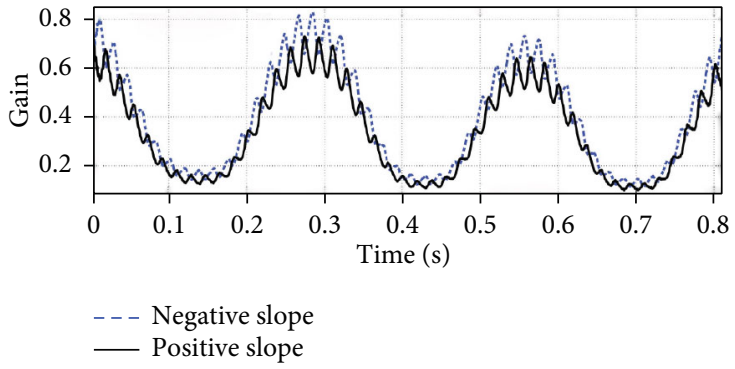

(a)

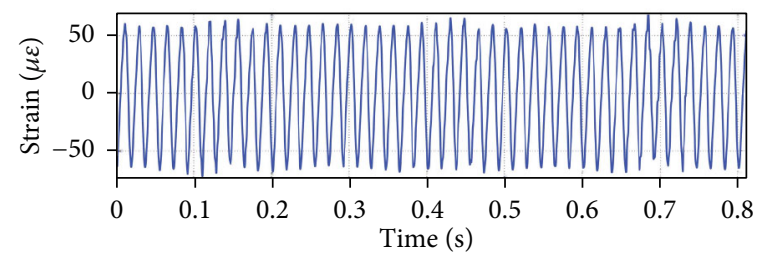

(b)

Figure 3: (a) Two SA-BOTDA gain measurements induced by the $55-\mathrm{Hz}$ strain vibrations, took at opposite slopes of the BGS in the presence of $>6 \mathrm{~dB}$ pump power variation. (b) The extracted $55 \mathrm{~Hz}$ strain variations using double-SA-BOTDA [31].

ratio (BPGR) has been proposed [32, 33], which combines both the Brillouin phase shift and Brillouin gain. Mathematically, the Brillouin phase spectrum (BPS) and BGS can be expressed as [32]

$$
\begin{aligned}
& \operatorname{BPS}\left(v-v_{B}(z), P(z)\right)=-K \cdot P(z) \cdot \frac{2 g_{0} \Delta v_{B}\left(v-v_{B}(z)\right)}{\left(\Delta v_{B}\right)^{2}+4\left(v-v_{B}(z)\right)^{2}}, \\
& \operatorname{BGS}\left(v-v_{B}(z), P(z)\right)=K \cdot P(z) \cdot \frac{g_{0}\left(\Delta v_{B}\right)^{2}}{\left(\Delta v_{B}\right)^{2}+4\left(v-v_{B}(z)\right)^{2}} .
\end{aligned}
$$

Then, the BPGR can be given by [32]

$$
\operatorname{BPGR}\left(v-v_{B}(z)\right)=\frac{B P S\left(v-v_{B}(z), P(z)\right)}{B G S\left(v-v_{B}(z), P(z)\right)}=-\frac{2\left(v-v_{B}(z)\right)}{\Delta v_{B}} .
$$

According to Equation (6), the BPGR is linearly proportional to the BFS $v_{B}(z)$; therefore, a much wider strain dynamic range can be realized. Moreover, the BPGR is independent on the pump power variation, which leads to a high measurement accuracy.

Figure 4(a) shows the detailed experimental setup. Different from the conventional SA-BOTDA system, a MachZehnder interferometer with an acousto-optic modulator (AOM) incorporated into its lower branch was employed to extract both the BPS and the BGS. When a periodical strain with a peak-to-peak value of $2000 \mu \varepsilon$ was applied to the FUT, the BFS change measured using Brillouin phase shift and BPGR are given in Figures 4(b) and 4(c), which shows that the strain dynamic range is enlarged significantly. By using the proposed system, a strain dynamic range of 100 $\mathrm{MHz}(2000 \mu \varepsilon)$, a sampling rate of $1 \mathrm{kHz}$, a spatial resolution 


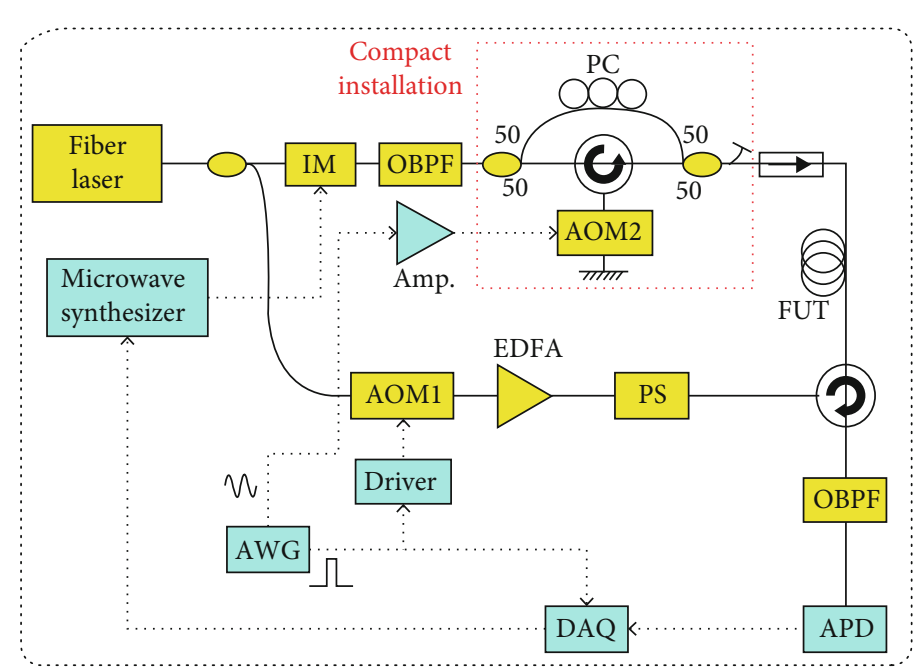

(a)

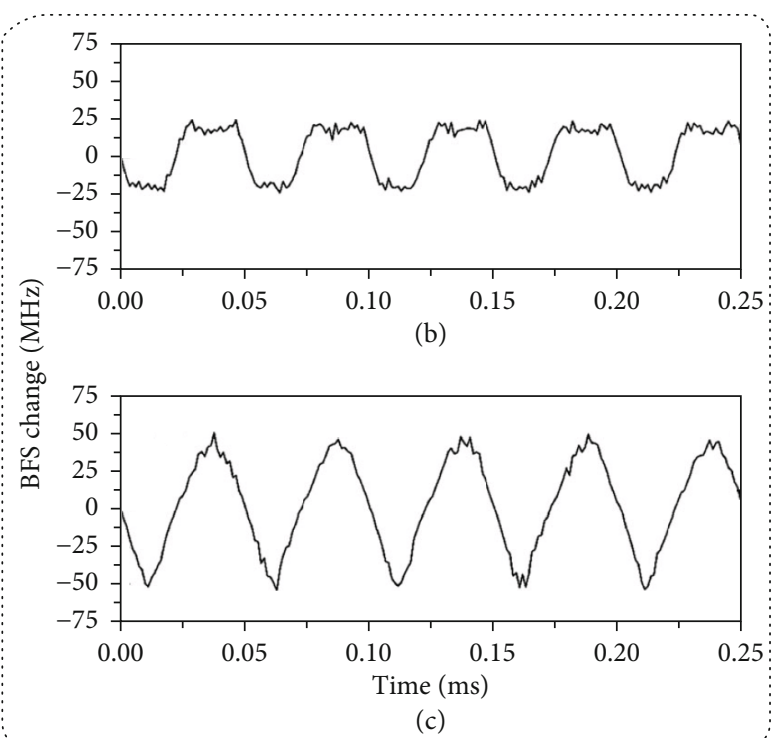

FIGURE 4: (a) SA-BOTDA experimental setup. IM: intensity modulator; PC: polarization controller; AOM: acousto-optic modulator; EDFA: erbium-doped fiber amplifier; PS: polarization scrambler; FUT: fiber under test; AWG: arbitrary waveform generator; APD: avalanche photodetector; OBPF: optical bandpass filter; DAQ: data acquisition system. (b, c) The BFS change measured by using Brillouin phase shift and Brillouin phase-gain ratio [32].

of $2.5 \mathrm{~m}$, and a measurement range of $2 \mathrm{~km}$ were achieved with good immunity to pump power variation.

3.1.4. Multi-SA-BOTDA. In some applications where large strain measurement is required, a much wider strain dynamic range is required. To fulfill the requirement, $\mathrm{Ba}$ et al. proposed a multi-SA-BOTDA technique in 2016 [34]. As shown in Figure 5(a), the left part denotes the doubleSA-BOTDA, and the right part denotes the multi-SABOTDA. In the multi-SA-BOTDA, the probe wave consists of several frequency tones, which is realized using a frequency-agile modulation technique. When the BFS is between Tone 1 and Tone 2, it can be demodulated by the gains of Tone 1 and Tone 2 using the same algorithm as double-SA-BOTDA. When the BFS is between Tone 2 and Tone 3, these two tones can be utilized to extract the BFS. In this way, the BFS can always be determined via a pair of neighboring tones. Since the frequency differences between the probe tones and the pump are known, the BFS can be demodulated accurately. It is worth noting that when a probe wave with $N$ frequency tones is employed, the strain dynamic range can be enlarged by a factor of $N-1$, while the sampling rate is reduced by $N$ times compared with the conventional SA-BOTDA.

The experimental setup of the multi-SA-BOTDA is similar to the conventional SA-BOTDA system, except for an AWG employed to generate complex probe wave with several frequency tones. In this work, to make the comparison more reliable, two different methods including the multiSA-BOTDA and the curve-fitting-based fast BOTDA were used to track a nonsine-changed dynamic strain applied to the FUT, and the experimental results are shown in Figure 5(b). The results obtained using these two methods matched well. By using the proposed technique, an ultrawide strain dynamic range of $241 \mathrm{MHz}(5000 \mu \varepsilon)$, a sampling rate of $1 \mathrm{kHz}$, a spatial resolution of $1 \mathrm{~m}$, and a measurement range of $32 \mathrm{~m}$ were achieved.

Subsequently, to further improve the performance of the sensing system, Zhou et al. proposed a novel technique by combining the BPGR concept and the multislope-assisted measurement scheme [33], and an ultrawide strain dynamic range of $254.3 \mathrm{MHz}(5372.9 \mu \varepsilon)$ was realized.

In the abovementioned multi-SA-BOTDA systems, the frequency of the probe wave is switched rapidly using a frequency agile modulation technique, where a wide strain dynamic range is achieved at the expense of a reduced sampling rate. Recently, Zheng et al. proposed a novel coherent multi-SA-BOTDA [35], and all the slopes can be interrogated simultaneously; therefore, a large dynamic range can be attained without frequency sweep.

Figure 6(a) shows the experimental setup. An AOM at the lower branch was employed to generate the pulsed pump wave. On the upper branch, an electro-optic modulator (EOM) driven by an $11-\mathrm{GHz}$ RF signal along with a fiber Bragg grating (FBG) was employed to generate a downshifted lightwave. Subsequently, another EOM (working frequency: $60 \mathrm{MHz}$ ) and AOM (working frequency: $240 \mathrm{MHz}$ ) connected after the FBG were used to synthesize the multitone probe wave and the reference wave. The frequency spectrum of the probe wave is shown in the inset of Figure 6(a). In this way, all the Brillouin gain slopes and phase-shift slopes can be extracted simultaneously from the low-frequency beat signals at different frequencies. Figure 6(b) shows the measured dynamic strains when the fiber segment is stretched by an electrical motor with different frequencies. By using this method, a strain dynamic range of $180 \mathrm{MHz}$, a sampling rate of $1.5 \mathrm{kHz}$, and a spatial resolution of $2.5 \mathrm{~m}$ over a measurement range of $2 \mathrm{~km}$ were achieved with good immunity to pump power variation. 


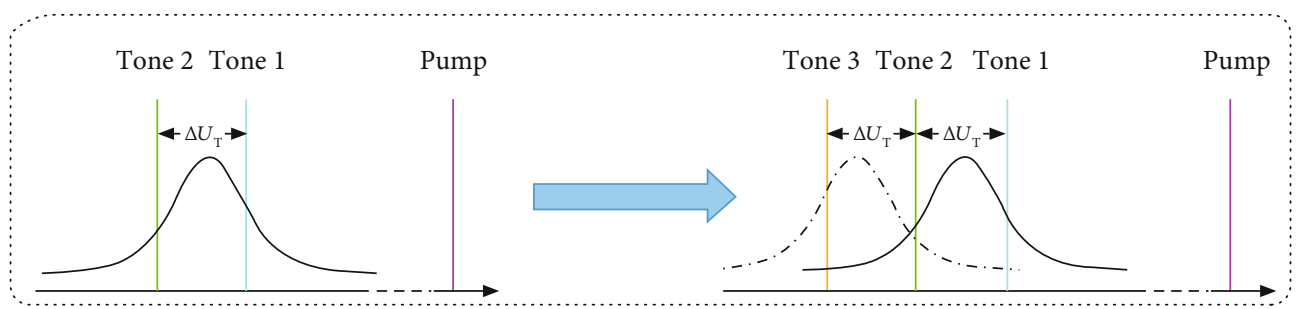

(a)

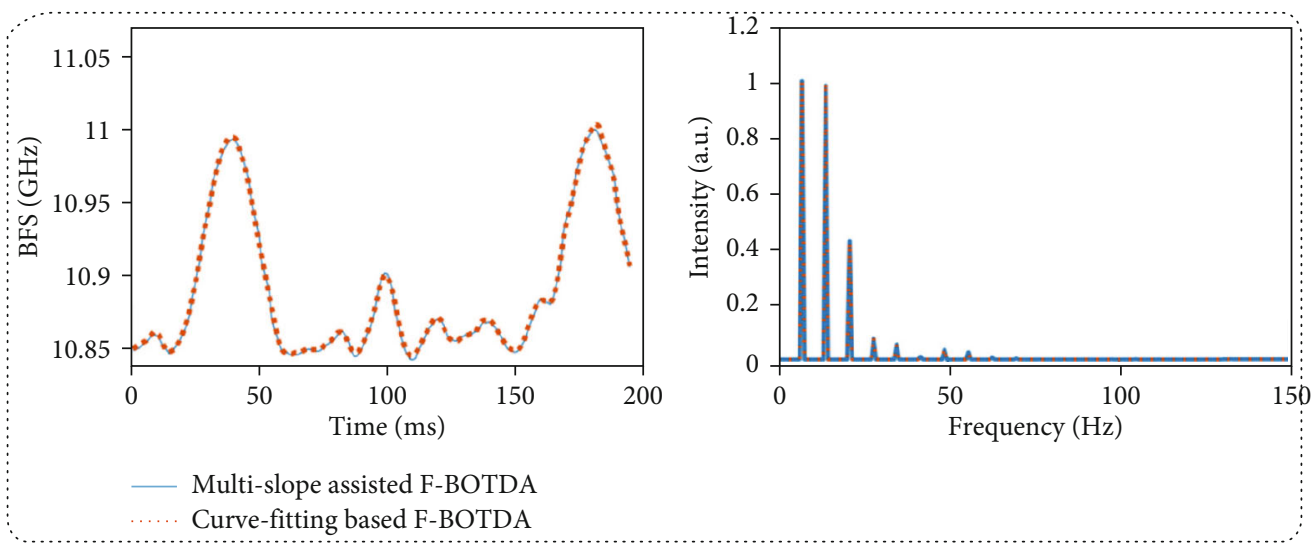

(b)

FIGURE 5: (a) Schematic illustration of multi-SA-BOTDA. (b) The track of BFS in time-domain and its power spectrum when a nonsinechanged dynamic strain is applied to the FUT [34].

3.1.5. SA-BOTDA Enhanced by BGS Engineering. BGS engineering is another technique to improve the performance of SA-BOTDA. In 2018, we proposed an SA-BOTDA with an enlarged strain dynamic range by manipulating the BGS shape [40]. In the proposed method, the pump wave (wP) was firstly modulated by two-tone RF signals $\left(\omega_{\mathrm{RF}}\right.$ and $\left.2 \omega_{\mathrm{RF}}\right)$ and then carved to an optical pulse, and the modulated light wave can be expressed as

$$
\begin{aligned}
E_{\text {Pump }}(t)= & A(t) \cdot\left[1+A_{1} \cos \left(\omega_{\mathrm{RF}} t+\varphi_{1}\right)\right. \\
& \left.+A_{2} \cos \left(2 \omega_{\mathrm{RF}} t+\varphi_{2}\right)\right] \cdot \exp \left(j \omega_{P} t\right),
\end{aligned}
$$

where $A(t)$ is the envelope of the pump pulse, $A_{1}$ and $A_{2}$ are the modulation depths of the two tones, respectively, and $\varphi_{1}$ and $\varphi_{2}$ are the corresponding modulation phases. By properly controlling the modulation frequency and depths, the BGS can be reconfigured to a desired shape with a wide linear slope range, which leads to an enlarged strain dynamic range.

The experimental setup is shown in Figure 7(a), where an intensity modulator (IM) and an AOM were used jointly to generate the complex optical pump pulse. BGSs obtained using different pump pulses are shown in Figure 7(b). It can be observed that when two-tone RF signals were utilized, the linear slope range of the BGS was enlarged significantly. Figure 7 (c) shows the time-domain waveform and the power spectrum of the measured sinusoidal dynamic strains using different methods. By using the proposed technique, a strain dynamic range of $100 \mathrm{MHz}(2000 \mu \varepsilon)$, a sampling rate of $1 \mathrm{kHz}$, a measurement accuracy of $5.26 \mu \varepsilon$, and a spatial res- olution of $2.5 \mathrm{~m}$ over a measurement range of $400 \mathrm{~m}$ were achieved.

Recently, to improve the sensitivity of SA-BOTDA, Feng et al. proposed a novel BGS engineering technique by combining the Brillouin gain and loss [42]. To synthesize the Brillouin spectrum with a desired shape, two probe waves with carefully-designed frequencies were employed, where one generated a Brillouin gain and the other one introduced a Brillouin loss. By using this technique, a much wider drift tolerant frequency range (DTFR) and a measurement accuracy of $8.8 \mu \varepsilon$ were achieved, and the sampling rate was as high as $12.5 \mathrm{kHz}$ at the expense of a limited measurement range.

3.2. SA-BOTDR. BOTDA based on SBS provides a high single-to-noise ratio (SNR) and measurement accuracy, but it requires two-end access of optical fibers to make possible the interaction between the two counterpropagating lightwaves. Alternately, BOTDR, as a singleended monitoring system, has a great potential in industrial applications due to its high flexibility and robustness in practical implementation and maintenance, because if the fiber is broken, measurement can still be made up to the breaking point.

To improve the measurement speed of BOTDR, Maraval et al. proposed a novel SA-BOTDR technique for singleended truly distributed dynamic strain measurement in 2018 [42]. In this system, the optical local oscillator frequency is carefully adjusted to measure the backscattered Brillouin signal power at the maximum slope of the BGS 


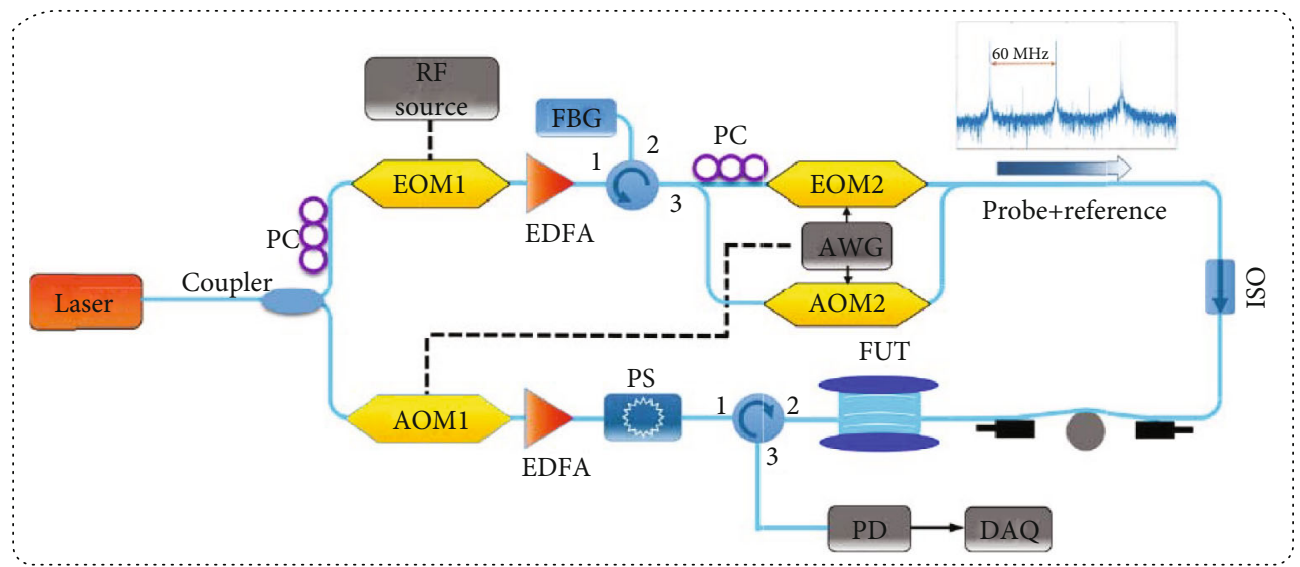

(a)

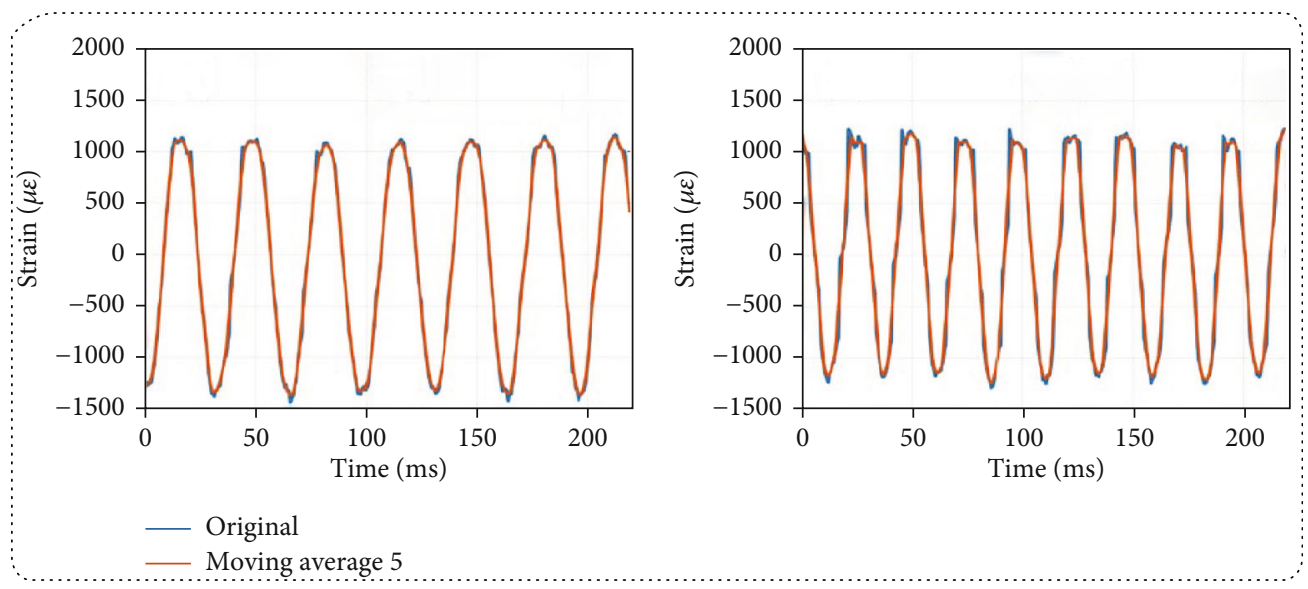

(b)

FIgURE 6: (a) Multi-SA-BOTDA experimental setup. PC: polarization controller; RF: radiofrequency; EOM: electro-optic modulator; EDFA: erbium-doped fiber amplifier; FBG: fiber Bragg grating; AOM: acousto-optic modulator; AWG: arbitrary waveform generator; PS: polarization scrambler; ISO: isolator; PD: photodetector; DAQ: data acquisition card (inset: the frequency spectrum of the probe wave). (b) Measured dynamic strains when the fiber segment are stretched by an electrical motor with different frequencies [35].

along the FUT. In this way, strain-induced BFS change will be detected by amplitude variations. Figure $8(\mathrm{a})$ shows the experimental setup, and a 10.4-m-long steel pipe was used as the demonstrator, where optical fibers were adhered on its surface. Figure 8(b) shows the measured displacement variations at the central position of the steel pipe when it is oscillated with a frequency of $1.1 \mathrm{~Hz}$, which matches well with the results obtained by the laser sensor. The sampling rate of the SA-BOTDR was set to be $7.6 \mathrm{~Hz}$. Similar to the conventional SA-BOTDA system, the strain dynamic range of SA-BOTDR is limited to $30 \mathrm{MHz}(600 \mu \varepsilon)$, and the sampling rate is about tens of $\mathrm{Hz}$, which is limited by the low SNR of the BOTDR system.

3.3. Slope-Assisted Brillouin Dynamic Grating Sensor. In SABOTDA and SA-BOTDR, the spatial resolution is determined by the duration of the pump pulse, which is practically limited to $10 \mathrm{~ns}$-equivalent to $1 \mathrm{~m}$ spatial resolution-since the broadening of the pump spectrum that results from a decreased pulse duration leads to a significant spectral spreading of the measured BGS. To improve the spatial resolution, Bergman et al. proposed a novel dynamic and distributed slope-assisted sensing system based on the BDG in a polarization-maintaining fiber (PMF) [43-45]. As shown in Figure 9(a), BDGs are generated in a PMF by two counter-propagating optical pump waves ( $\omega_{\text {Pump- } H}$ and $\omega_{\text {Pump-L }}$, where $\left.\omega_{\text {Pump-H }}>\omega_{\text {Pump-L }}\right)$, which are copolarized along the slow axis of the PMF. The interference between these two optical pump waves generates a moving longitudinal density wave, whose strength depends on the frequency interval between two pumps. Via the elastooptic effect, this induced longitudinal acoustic wave is actually a moving Bragg grating, which can back-reflect an orthogonally polarized probe pulse $\left(\omega_{\text {Probe }}\right)$, and the reflectivity $R_{\text {Probe }}$ depends on the relationship among the three optical frequencies involved: $\omega_{\text {Pump- } H}, \omega_{\text {Pump-L}}$, and $\omega_{\text {Probe }}$, attaining a maximum when the phase-matching conditions are met, given by $v_{B}=\omega_{\text {Pump }-H}-\omega_{\text {Pump }-L}, v_{\mathrm{BDG}}=\omega_{\text {Probe }}-$ $\omega_{\text {Pump-H}}$, where $v_{B}$ is the BFS of the PMF, and $v_{\mathrm{BDG}}$ is the BDG frequency shift, which is determined by the birefringence of the PMF.

According to the theoretical analysis, the reflectivity $R_{\text {Probe }}$ is dependent on $v_{B}$ and $v_{\mathrm{BDG}}$, which are functions of 


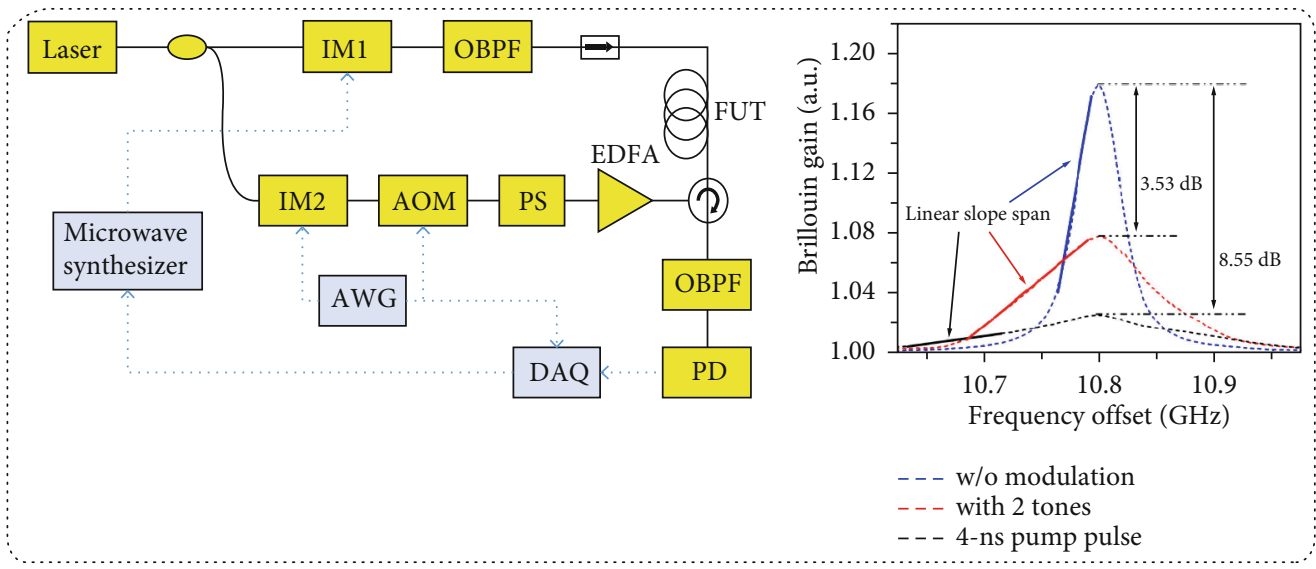

(a)

(b)

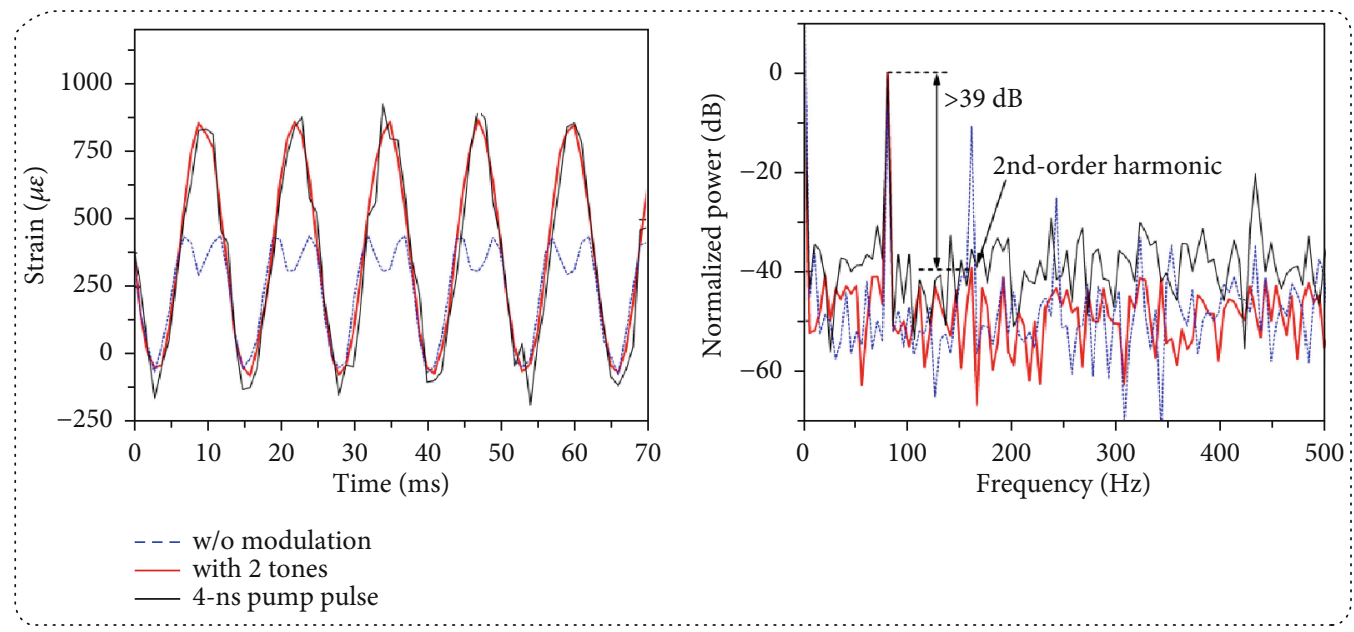

(c)

FIGURE 7: (a) SA-BOTDA experimental setup. IM: intensity modulator; OBPF: optical band-pass filter; FUT: fiber under test; AOM: acoustooptic modulator; PS: polarization scrambler; EDFA: erbium-doped fiber amplifier; AWG: arbitrary waveform generator; DAQ: data acquisition system; PD: photo-detector. (b) BGSs obtained using different pump pulses. (c) The time-domain waveform and the power spectrum of the measured dynamic strains [40].

the strain applied to the optical fiber. Therefore, by carefully setting the values of $\omega_{\text {Pump-H}}, \omega_{\text {Pump-L }}$, and $\omega_{\text {Probe }}$, the strain information can be extracted from the power variations of the reflected probe wave. It is worth noting that the generated BDG is practically independent on the duration of the probe pulse; therefore, a much higher spatial resolution can be achieved. The detailed experimental setup is shown in Figure 9(a), and two laser diodes were employed to generate the pump and probe waves. Figure 9(b) shows the measured dynamic strains when a sinusoidal vibration with a frequency of $400 \mathrm{~Hz}$ was applied to the FUT. By using the proposed technique, an ultrahigh sampling rate of $16 \mathrm{kHz}$ and a high spatial resolution of $4.2 \mathrm{~cm}$ over a measurement range of $5 \mathrm{~m}$ were realized. Subsequently, a probe coding technique was introduced into the slopeassisted BDG sensing system by the same group [44], to improve the sensitivity and the sampling rate further, and an ultrahigh sampling rate of $1 \mathrm{MHz}$ along with a spatial resolution of $20 \mathrm{~cm}$ was achieved.

\section{Slope-Assisted Brillouin Correlation Domain Sensors}

Researchers have proposed two kinds of slope-assisted Brillouin correlation domain sensing systems, including SABOCDA [11, 27, 28, 46-48] and SA-BOCDR [49-58].

4.1. SA-BOCDA. Unlike time-domain Brillouin sensors, where a pulsed pump is utilized to achieve distributed measurement, in BOCDA, continuous-wave pump and probe are injected into the FUT from opposite directions. Both the pump and the probe are sinusoidally frequency-modulated to generate position-dependent Brillouin interaction along the FUT. At specific positions, called correlation peaks $(\mathrm{CPs})$, the pump-probe frequency interval remains unchanged, leading to a strong SBS interaction. While at other positions, the pump-probe frequency interval changes randomly, leading to a weak SBS interaction. In this way, distributed optical fiber sensing with a spatial resolution as high as a 


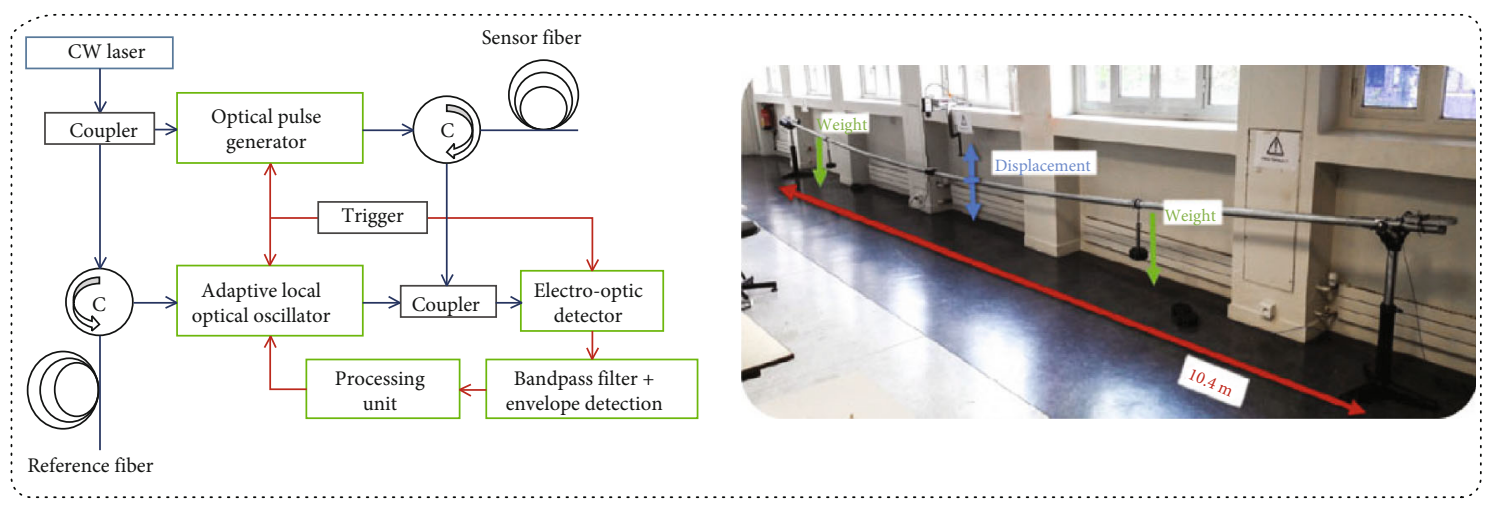

(a)

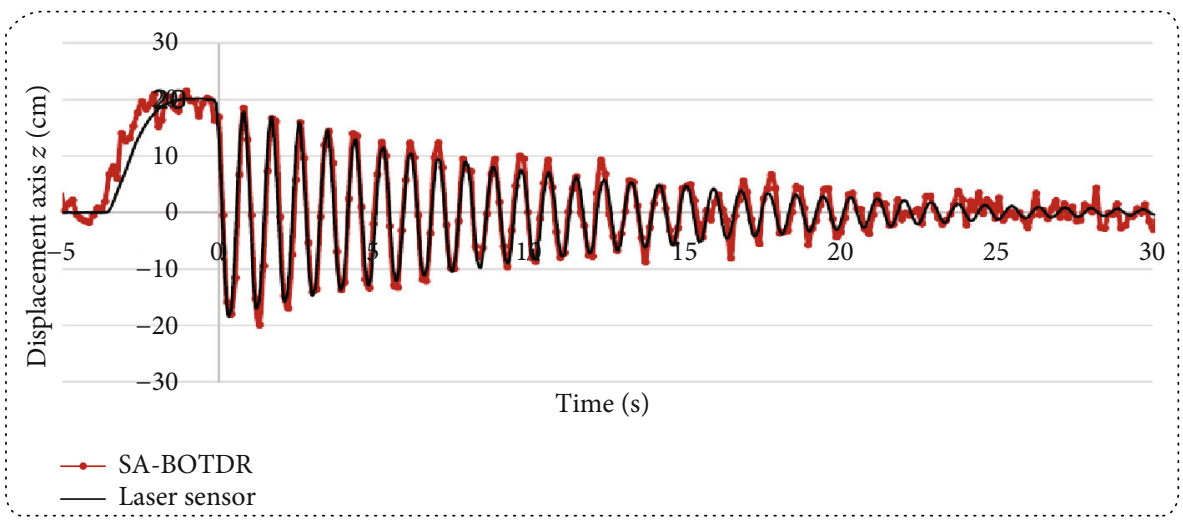

(b)

FIGURE 8: (a) SA-BOTDR experimental setup and the photo of the demonstrator: 10.4-m-long steel pipe with optical fibers adhered. (b) Measured displacement variations at the central position of the steel pipe when it is oscillated with a frequency of $1.1 \mathrm{~Hz}$ [42].

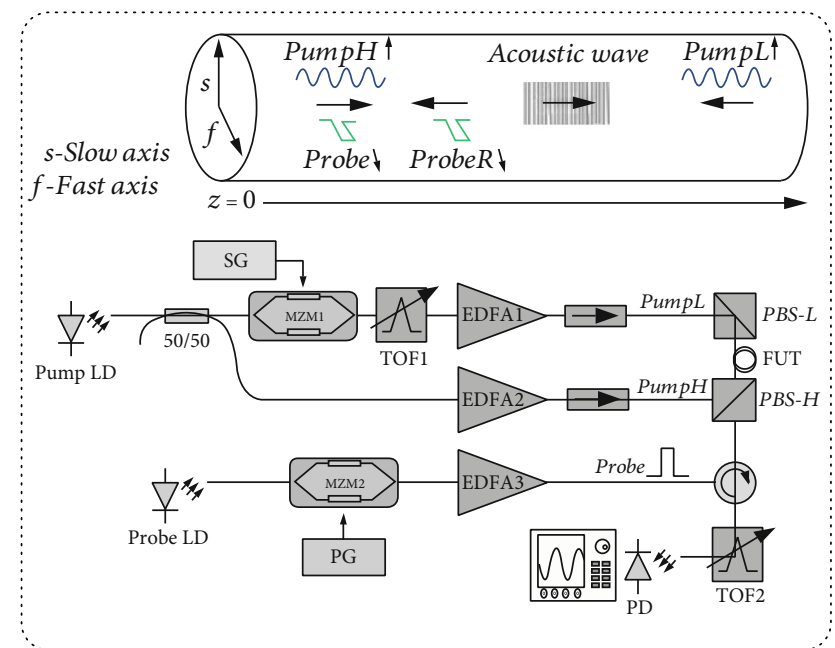

(a)

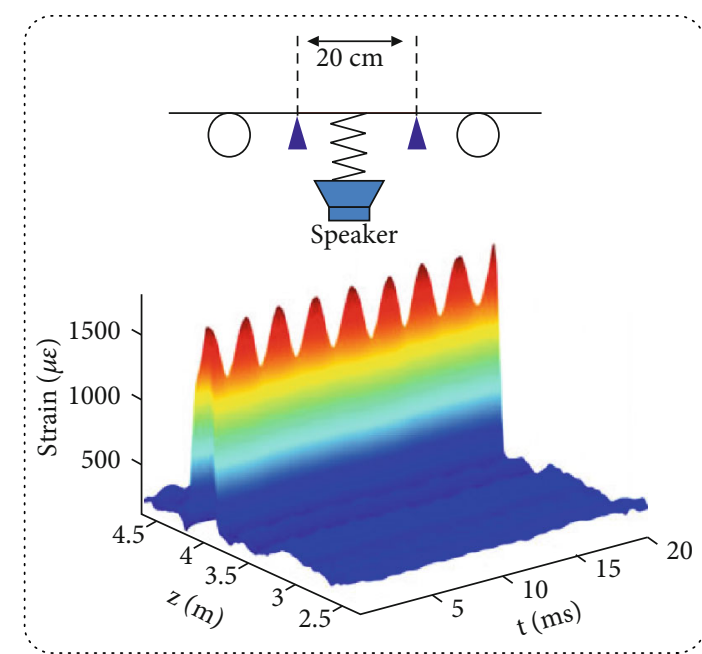

(b)

FIGURE 9: (a) Experimental setup of slope-assisted sensing system based on BDG. LD: laser diode; SG: signal generator; MZM: Mach-Zehnder modulator; TOF: tunable optical filter; EDFA: erbium-doped fiber amplifier; PG: pulse generator; PBS: polarization beam splitter; FUT: fiber under test; PD: fast photodiode. (b) Measured dynamic strains when a 20-cm-long fiber section was disturbed [43].

few millimeters has been achieved. Up to now, several techniques have been proposed to improve the performance of $\mathrm{SA}-\mathrm{BOCDA}$ in terms of sampling rate, strain dynamic range, immunity to pump power variation, and number of effective sensing points, mainly including the double-SA-BOCDA and the SA-BOCDA based on chaotic laser. 


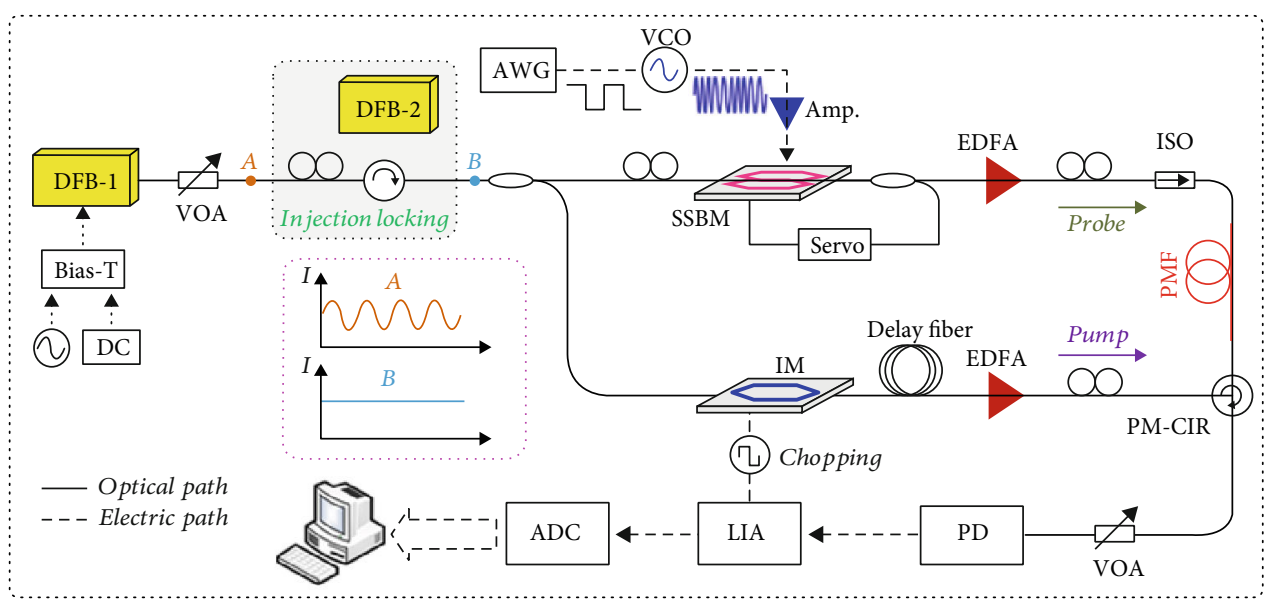

(a)

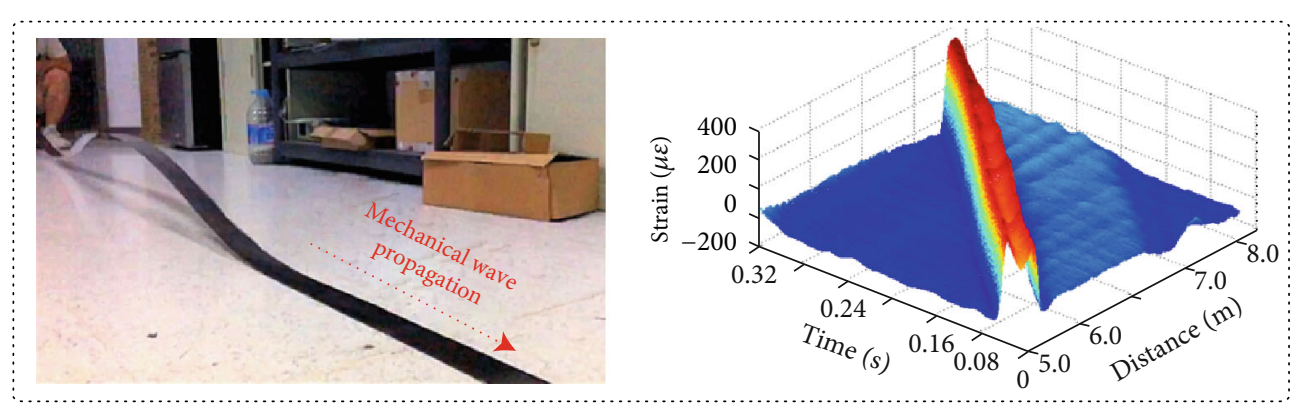

(b)

Figure 10: (a) Double-SA-BOCDA experimental setup. DFB-LD: distributed feedback laser diode; VOA: variable optical attenuator; SSBM: single-sideband modulator; RF: radio-frequency signal generator; EDFA: Erbium-doped fiber amplifier; PMF: polarization-maintaining fiber; IM: intensity modulator; PD: photodetector; LIA: lock-in amplifier; ADC analog-to-digital converter. (b) Measured strain distribution as a function of both time and distance when the mechanical wave is propagated along the FUT $[27,28]$.

4.1.1. Double-SA-BOCDA. The slope-assisted measurement scheme was firstly introduced into the BOCDA system by Hotate et al. in 2003 [11], and dynamic strain measurement was implemented at a fixed sensing point. In 2017, the double-slope-assisted measurement scheme was introduced into the phase-BOCDA system by Morosi et al., and distributed static strain measurement with a spatial resolution of 2 $\mathrm{cm}$ and good immunity to pump power variation was realized [46]. In 2019, we proposed a novel double-SA-BOCDA based on synthesis of optical coherence function (SOCF) $[27,28]$. The experimental setup of the proposed system is shown in Figure 10(a). The laser source was sinusoidally frequency modulated to synthesize periodical correlation peaks, thus to implement distributed measurement. An injectionlocking technique was employed to remove the parasitic intensity noise of the laser source, so as to improve the measurement accuracy and the stability of the system. A PMF was used as the FUT to avoid the polarization-dependent power variations. A lock-in amplifier- (LIA-) based detection technique was also introduced to improve the system SNR.

As a demonstration, the proposed system was used to track the propagation of mechanical wave along the FUT. A 3.0-m-long $(5.0 \mathrm{~m}-8.0 \mathrm{~m})$ fiber section was tightly adhered on the top surface of a thick rubber sheet. A mechanical wave is manually generated and then propagated along the FUT.
The measured temporal variations of strain distribution are shown in Figure 10(b), and the propagation speed was calculated to be $9 \mathrm{~m} / \mathrm{s}$, which matches well with the results measured by SA-BOTDA [29]. Moreover, benefiting from the ultrahigh spatial resolution, it is found that the bandwidth of the mechanical wave becomes narrower when it propagates from $5.0 \mathrm{~m}$ to $8.0 \mathrm{~m}$. By using the proposed technique, a sampling rate of $625 \mathrm{~Hz}$, a strain dynamic range of 35 $\mathrm{MHz}(700 \mu \varepsilon)$, and a spatial resolution of $7 \mathrm{~cm}$ over a measurement range of $10 \mathrm{~m}$ were achieved with good immunity to fiber loss and pump power variation.

4.1.2. SA-BOCDA Based on Chaotic Laser. In the SOCF-based BOCDA system, there is a trade-off between the measurement range and the spatial resolution, and the number of effective sensing points is normally limited to about 1000. Recently, Zhang et al. proposed a novel Brillouin correlation domain sensing system by using a chaotic laser as the optical source of the system, which is called chaotic-BOCDA [19]. Moreover, to improve the measurement speed, the slope-assisted measurement scheme was introduced into this new type of BOCDA sensor [47]. It is worth noting that the linear slope range of the BGS was broadened since the broadband chaos provides a Gaussian-shape pump-probe beat spectrum, and therefore, the strain dynamic range was extended accordingly. 


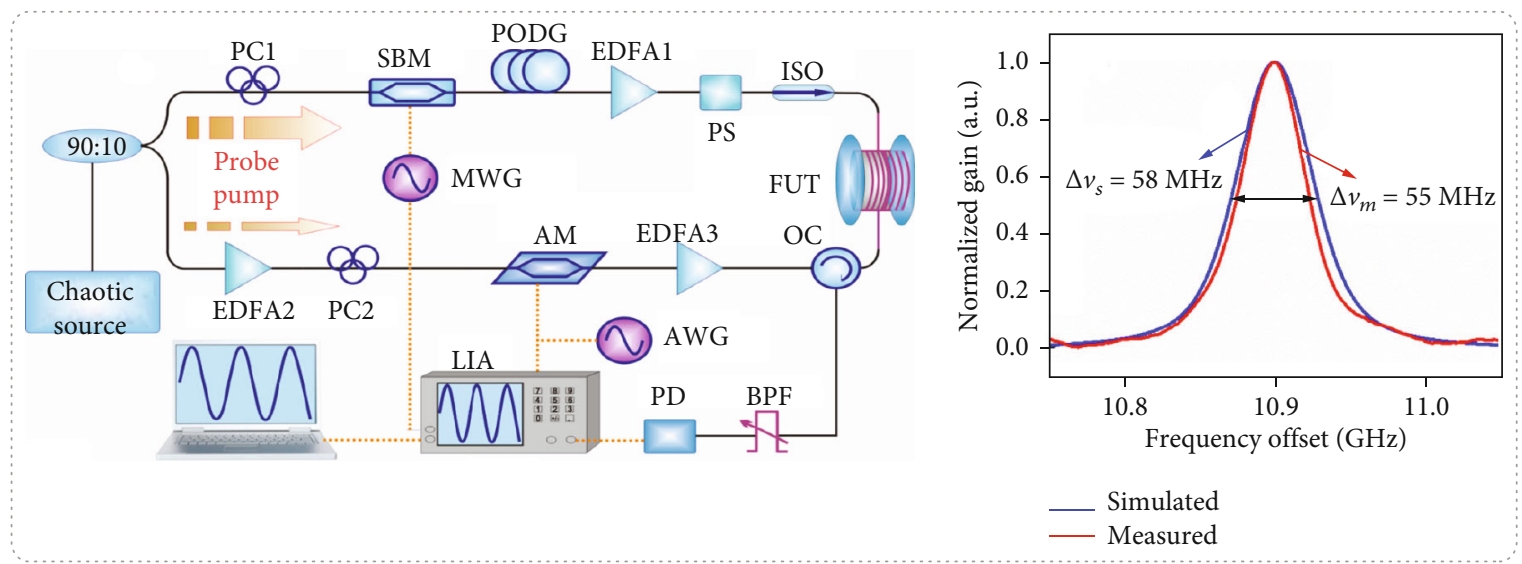

(a)
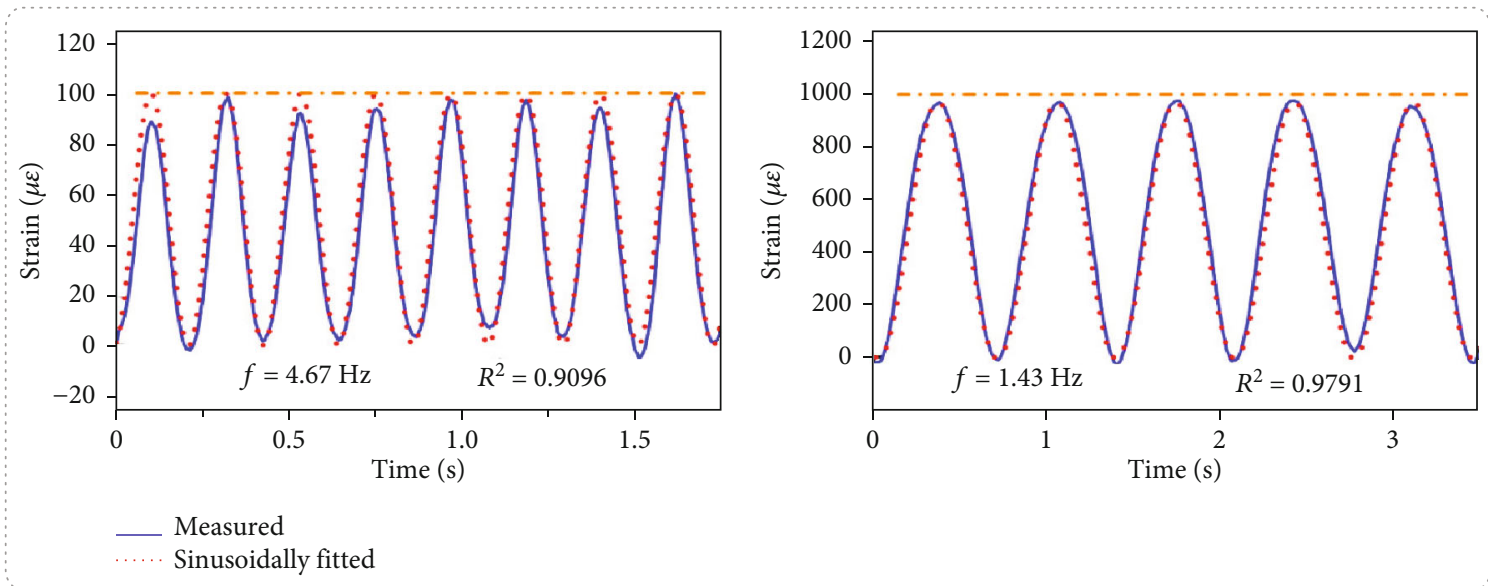

(b)

FIGURE 11: (a) Experimental setup of the slope-assisted chaotic BOCDA and the measured BGS. (b) Measured time traces when sinusoidal dynamic strains with peak-to-peak values of $100 \mu \varepsilon$ and $1000 \mu \varepsilon$ were applied [47].

Figure 11(a) shows the experimental setup of the slope-assisted chaotic BOCDA. The output of the chaotic laser was divided into two portions, where one portion was used as the pump, and the other one was used as the probe. A programmable optical delay generator (PODG) was used to localize the sensing position precisely and to implement distributed measurements. The FWHM of measured BGS was around $55 \mathrm{MHz}$, which matches well with the simulations results. In dynamic measurements, the FUT was stretched periodically via an electric-driven motor. Figure 11(b) shows the measured time traces when sinusoidal dynamic strains with peak-to-peak values of $100 \mu \varepsilon$ and $1000 \mu \varepsilon$ were applied, and no obvious distortion was observed. By using the slope-assisted chaotic BOCDA, a strain dynamic range of $55 \mathrm{MHz}(1200 \mu \varepsilon)$ and a spatial resolution of $3.45 \mathrm{~cm}$ over a measurement range of $130 \mathrm{~m}$ were realized. Dynamic strains with frequencies up to $4.67 \mathrm{~Hz}$ was measured, but distributed dynamic measurement was yet to be demonstrated. Recently, the same group has also analyzed the effect of chaotic time delay signature (TDS) on the measured BGS in the slope-assisted chaotic BOCDA [48]. By optimizing the parameters of the chaotic laser and eliminating the
TDS, BGS without subpeak was obtained, and dynamic strains with frequency of $1.17 \mathrm{~Hz}$ were measured in a fully distributed manner.

4.2. SA-BOCDR. BOCDR is another important Brillouin sensing technique since it provides both high spatial resolution and single-end-access capability. In BOCDR, to spatially resolve the sensing locations, the laser source of the system is sinusoidally frequency-modulated to generate periodical CPs in the FUT, which is similar to BOCDA. The reference wave and the Stokes wave are combined in an optical coupler and, then, detected by a high-sensitivity photodiode (PD). Since the SpBS signal is used for sensing in BOCDR, it provides a unique advantage of single-end-access to FUT and shows good flexibility. In 2016, Mizuno et al. firstly introduced the slope-assisted measurement scheme into the BOCDR system to improve its measurement speed [49]. Similar to SABOTDR, in SA-BOCDR. the frequency interval between the pump wave and the reference (local) wave was set at the linear slope of the BGS, and the temperature/strain information was extracted from the Brillouin signal power variations.

The experimental setup of SA-BOCDR is shown in Figure 12(a). The sinusoidally frequency-modulated 


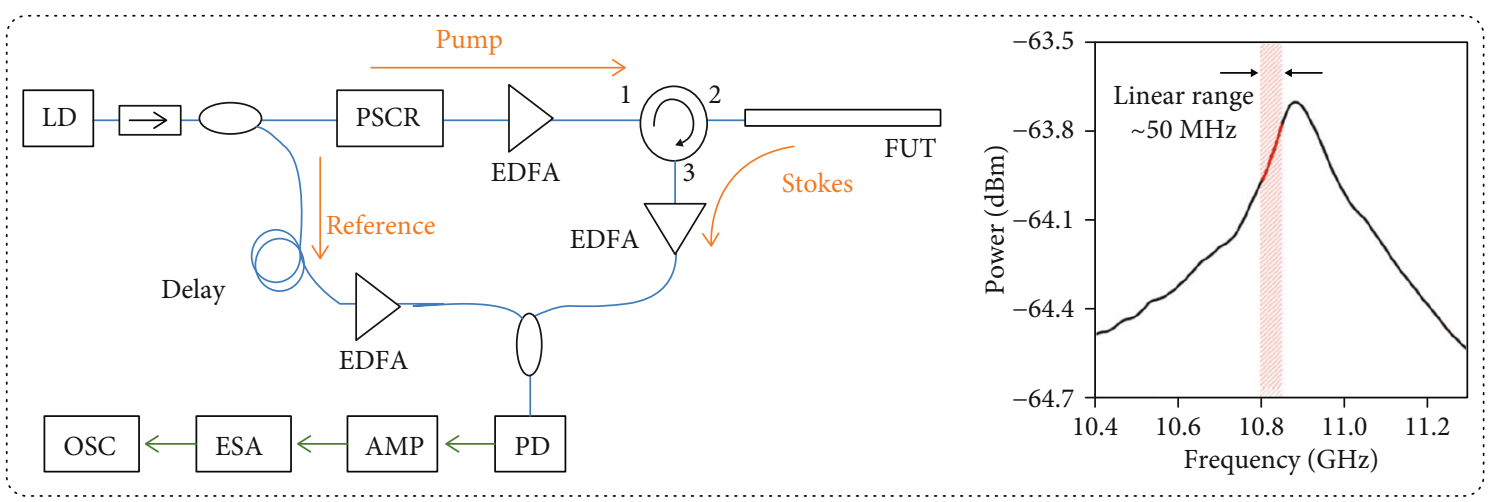

(a)
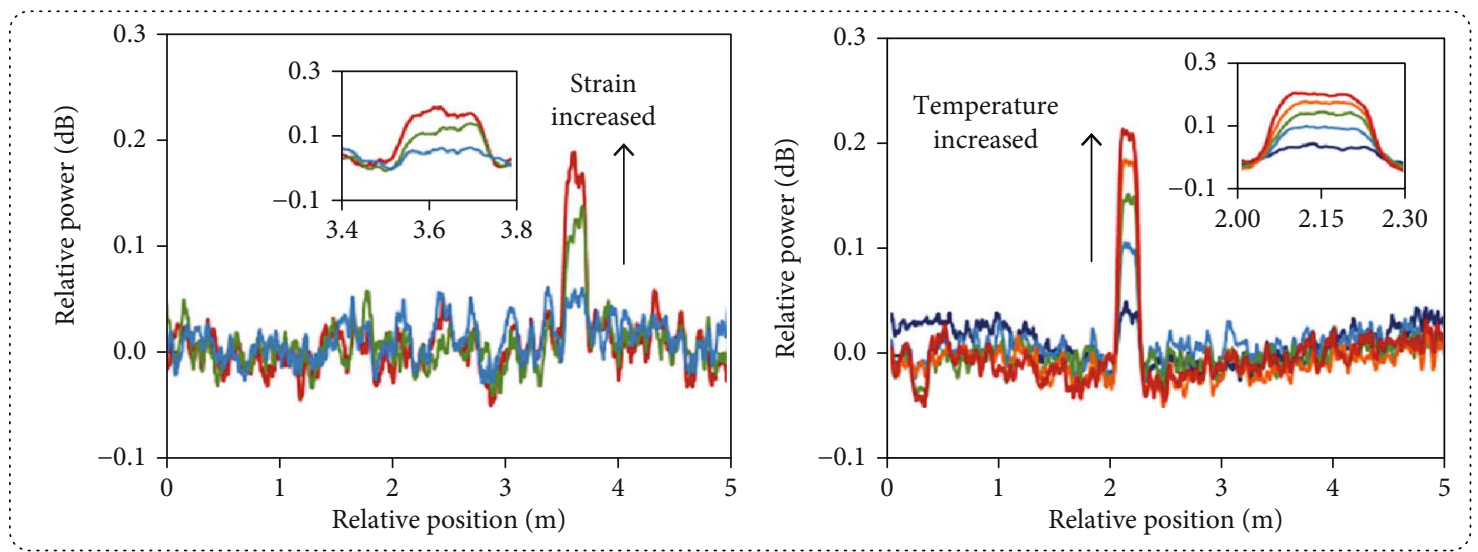

(b)

FIGURE 12: (a) Experimental setup of SA-BOCDR. LD: laser diode; PSCR: polarization scrambler; EDFA: erbium-doped fiber amplifier; FUT: fiber under test; PD: photodiode; AMP: amplifier; ESA: electrical spectrum analyzer; OSC: oscilloscope. (b) Measured Brillouin power change distribution along the FUT when strains were locally applied or temperature was changed [49].

lightwave generated by the laser diode was divided into two portions by an optical coupler, one portion was used as the pump wave, and the other one was used as the reference wave. A polarization scrambler was used to suppress the polarization-induced Brillouin power variations. The backscattered Stokes wave was combined with the reference wave by another optical coupler and, then, detected by a highspeed photodetector. The measured BGS is shown in the right part of Figure 12(a), and a linear slope range of 50 $\mathrm{MHz}$ was realized. Figure 12(b) shows the measured Brillouin power change distribution along the FUT when strains were locally applied or temperature was changed. It can be observed that the stretched of heated fiber section can be recognized clearly. By using the SA-BOCDR system, a spatial resolution of $8.8 \mathrm{~cm}$ and a strain dynamic range of $1000 \mu \varepsilon$ were realized with capability of single-end access. However, due to the low system SNR, the measurement accuracy of the system was relatively poor, and dynamic measurement was yet to be demonstrated.

Subsequently, great efforts have been made by the same research group to improve the performance of SA-BOCDR. In 2016, the relationship between the power-change distribution of SA-BOCDR and the actual BFS distribution along the FUT was theoretically and experimentally investigated [50], and a unique "beyond-nominal-resolution" effect was found, which has potential to improve the system spatial resolution further. Subsequently, various special optical fibers, including polymer optical fiber (POF) [51], bending-insensitive fiber [54], and PMF [57], were employed as the FUT in SABOCDR to improve its performance in terms of sensitivity, stability, and so on. The trade-off relationship between the strain dynamic range and the spatial resolution in SABOCDR was also investigated [56].

\section{Discussion}

Slope-assisted Brillouin-based fiber sensing is a powerful tool for structural health monitoring since it can provide continuous temperature and strain information along the whole fiber used for sensing applications in a fully real-time manner. Table 1 gives a comprehensive comparison among different slope-assisted Brillouin-based distributed fiber-optic sensors. It is clear that SBS-based sensors including SABOTDA, slope-assisted BDG sensor, and SA-BOCDA provide high performance in terms of sampling rate, measurement range, and measurement accuracy, but they require two-end access to the FUT. On the contrary, SpBS-based sensors including SA-BOTDR and SA-BOCDR has a unique advantage of single-end access to the FUT, but their performance is relatively poor due to the low system SNR. 


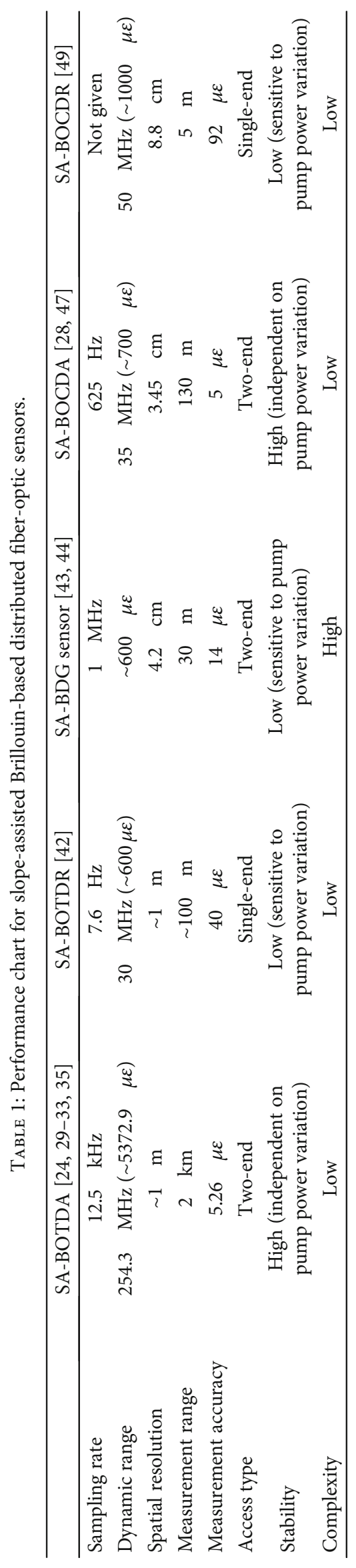


In future research, to further extend the application fields of slope-assisted Brillouin-based sensors, several potential research directions in this area are discussed: (1) the SA-BOTDA system provides a high sampling rate, a wide dynamic range, and a high measurement accuracy with low-complexity hardware, but its spatial resolution is limited to $1 \mathrm{~m}$ due to the limited lifetime of the phonon. Therefore, it is valuable to introduce advanced distributed measurement techniques, such as differential pulse-width pair (DPP) scheme [59,60], Brillouin echoes [61], and dark-pulse measurement scheme [62], into the SABOTDA system to improve its spatial resolution; (2) for SBSbased sensors, two-end access to the FUT is required to generate SBS interaction in the optical fiber. To improve the flexibility of the dynamic sensing system, single-end-access measurement techniques are desired to be proposed [63]; (3) for SpBS-based sensors, the measurement accuracy is relatively poor due to the low system SNR, which restricts its applications in real-world environments. Therefore, new techniques and concepts are in demand to improve the SNR of the sensing systems $[64,65]$.

\section{Conclusion}

This paper presents a comprehensive and systematic overview of the slope-assisted Brillouin-based distributed fiber-optic sensing techniques. The working principle and the state-ofthe-art of five kinds of slope-assisted Brillouin sensor are introduced, including SA-BOTDA, SA-BOTDR, slope-assisted BDG sensor, SA-BOCDA, and SA-BOCDR. Up to now, various techniques, including double-slope-assisted scheme, multislopeassisted scheme, and BGS engineering technique, have been proposed to improve the performance of the slope-assisted Brillouin sensor in terms of stability, strain dynamic range, sensitivity, and so on. It is anticipated that these distributed dynamic sensing systems will be commercialized and widely put into practice in the near future due to their unique advantages of high sampling rate, high spatial resolution, high measurement accuracy, and fully distributed measurement capability. The applications of the slope-assisted Brillouin-based distributed fiber-optic sensors in civil structural, aerospace, and power industry will finally make this technology beneficial to the society and ensure public security.

\section{Conflicts of Interest}

The authors declared that there is no conflict of interest regarding the publication of this article.

\section{Authors' Contributions}

X. Fan and Z. He create the outline of the manuscript. X. Fan, B. Wang, and G. Yang wrote the manuscript. X. Fan and Z. He revised the manuscript.

\section{Acknowledgments}

This work was supported by the National Science Foundation of China (NSFC) under Grant 61775132, 61735015, and 61620106015 .

\section{References}

[1] T. Horiguchi and M. Tateda, "BOTDA-nondestructive measurement of single-mode optical fiber attenuation characteristics using Brillouin interaction: theory," Journal of Lightwave Technology, vol. 7, no. 8, pp. 1170-1176, 1989.

[2] T. Kurashima, T. Horiguchi, and M. Tateda, "Distributedtemperature sensing using stimulated Brillouin scattering in optical silica fibers," Optics Letters, vol. 15, no. 18, pp. 1038-1040, 1990.

[3] T. Kurashima, T. Horiguchi, H. Izumita, S. Furukawa, and Y. Koyamada, "Brillouin optical-fiber time domain reflectometry," IEICE Transactions on Communications, vol. E76-B, no. 4, pp. 382-390, 1993.

[4] K. Shimizu, T. Horiguchi, and Y. Koyamada, "Measurement of distributed strain and temperature in a branched optical fiber network by use of Brillouin optical time-domain reflectometry," Optics Letters, vol. 20, no. 5, pp. 507-509, 1995.

[5] D. Garus, K. Krebber, F. Schliep, and T. Gogolla, "Distributed sensing technique based on Brillouin optical-fiber frequencydomain analysis," Optics Letters, vol. 21, no. 17, pp. 14021404, 1996.

[6] D. Garcus, T. Gogolla, K. Krebber, and F. Schliep, "Brillouin optical-fiber frequency-domain analysis for distributed temperature and strain measurements," Journal of Lightwave Technology, vol. 15, no. 4, pp. 654-662, 1997.

[7] R. Bernini, A. Minardo, and L. Zeni, "Distributed sensing at centimeter-scale spatial resolution by BOFDA: measurements and signal processing," IEEE Photonics Journal, vol. 4, no. 1, pp. 48-56, 2012.

[8] A. Minardo, R. Bernini, R. Ruiz-Lombera, J. Mirapeix, J. M. Lopez-Higuera, and L. Zeni, "Proposal of Brillouin optical frequency-domain reflectometry (BOFDR)," Optics Express, vol. 24, no. 26, 2016.

[9] K. Hotate, S. Ong, and S. Leng, "A correlation-based continuous-wave technique for measurement of dynamic strain along an optical fiber using brillouin scattering with fully distributed ability," in 2002 15th Optical Fiber Sensors Conference Technical Digest. OFS 2002(Cat. No.02EX533), Portland, OR, USA, May 2002.

[10] K. Hotate and M. Tanaka, "Distributed fiber Brillouin strain sensing with $1-\mathrm{cm}$ spatial resolution by correlation-based continuous-wave technique," IEEE Photonics Technology Letters, vol. 14, no. 2, pp. 179-181, 2002.

[11] K. Hotate and S. S. L. Ong, "Distributed dynamic strain measurement using a correlation-based Brillouin sensing system," IEEE Photonics Technology Letters, vol. 15, no. 2, pp. 272-274, 2003.

[12] Y. Mizuno, W. Zou, Z. He, and K. Hotate, "Proposal of Brillouin optical correlation-domain reflectometry (BOCDR)," Optics Express, vol. 16, no. 16, 2008.

[13] Y. Mizuno, W. Zou, Z. He, and K. Hotate, "Operation of Brillouin optical correlation-domain reflectometry: theoretical analysis and experimental validation," Journal of Lightwave Technology, vol. 28, no. 22, pp. 3300-3306, 2010.

[14] M. A. Soto, G. Bolognini, and F. D. Pasquale, "Optimization of long-range BOTDA sensors with high resolution using firstorder bi-directional Raman amplification," Optics Express, vol. 19, no. 5, pp. 4444-4457, 2011.

[15] Y. Dong, L. Chen, and X. Bao, "Time-division multiplexingbased BOTDA over 100km sensing length," Optics Letters, vol. 36, no. 2, pp. 277-279, 2011. 
[16] K. Song, Z. He, and K. Hotate, "Distributed strain measurement with millimeter-order spatial resolution based on Brillouin optical correlation domain analysis," Optics Letters, vol. 31, no. 17, pp. 2526-2528, 2006.

[17] R. Cohen, Y. London, Y. Antman, and A. Zadok, "Brillouin optical correlation domain analysis with 4 millimeter resolution based on amplified spontaneous emission," Optics express, vol. 22, no. 10, pp. 12070-12078, 2014.

[18] D. Ba, Y. Li, J. Yan, X. Zhang, and Y. Dong, "Phase-coded Brillouin optical correlation domain analysis with $2-\mathrm{mm}$ resolution based on phase-shift keying," Optics Express, vol. 27, no. 25, pp. 36197-36205, 2019.

[19] Y. Wang, M. Zhang, J. Zhang et al., "Millimeter-Level-SpatialResolution Brillouin optical correlation-domain analysis based on broadband chaotic laser," Journal of Lightwave Technology, vol. 37, no. 15, pp. 3706-3712, 2019.

[20] Y. Peled, A. Motil, and M. Tur, "Fast Brillouin optical time domain analysis for dynamic sensing," Optics Express, vol. 20, no. 8, pp. 8584-8591, 2012.

[21] D. Zhou, Y. Dong, B. Wang et al., "Single-shot BOTDA based on an optical chirp chain probe wave for distributed ultrafast measurement," Light: Science \& Applications, vol. 7, no. 1, 2018.

[22] V. L. Iezzi, S. Loranger, M. Marois, and R. Kashyap, "Highsensitivity temperature sensing using higher-order stokes stimulated Brillouin scattering in optical fiber," Optics Letters, vol. 39, no. 4, pp. 857-860, 2014.

[23] V. L. Iezzi, S. Loranger, and R. Kashyap, "High sensitivity distributed temperature fiber sensor using stimulated Brillouin scattering," Optics Express, vol. 25, no. 26, 2017.

[24] Y. Peled, A. Motil, L. Yaron, and M. Tur, "Slope-assisted fast distributed sensing in optical fibers with arbitrary Brillouin profile," Optics Express, vol. 19, no. 21, pp. 19845-19854, 2011.

[25] A. Motil, A. Bergman, and M. Tur, "State of the art of Brillouin fiber-optic distributed sensing," Optics \& Laser Technology, vol. 78, 2016.

[26] A. Kobyakov, M. Sauer, and D. Chowdhury, "Stimulated Brillouin scattering in optical fibers," Advances in Optics and Photonics, vol. 2, no. 1, pp. 1-59, 2010.

[27] B. Wang, X. Fan, and Z. He, "Distributed dynamic strain measurement with high repetition rate based on dual-slopeassisted BOCDA," in 2019 Asia Communications and Photonics Conference (ACP), Chengdu, China, November 2019.

[28] B. Wang, X. Fan, Y. Fu, and Z. He, "Distributed dynamic strain measurement based on dual-slope-assisted Brillouin optical correlation domain analysis," Journal of Lightwave Technology, vol. 37, no. 18, pp. 4573-4583, 2019.

[29] Y. Peled, A. Motil, I. Kressel, and M. Tur, "Monitoring the propagation of mechanical waves using an optical fiber distributed and dynamic strain sensor based on BOTDA," Optics express, vol. 21, no. 9, pp. 10697-10705, 2013.

[30] A. Minardo, G. Porcaro, D. Giannetta, R. Bernini, and L. Zeni, "Real-time monitoring of railway traffic using slope-assisted Brillouin distributed sensors," Applied Optics, vol. 52, no. 16, pp. 3770-3776, 2013.

[31] A. Motil, O. Danon, Y. Peled, and M. Tur, "Pump-power-independent double slope-assisted distributed and fast Brillouin fiber-optic sensor," IEEE Photonics Technology Letters, vol. 26, no. 8, pp. 797-800, 2014.

[32] G. Yang, X. Fan, and Z. He, "Strain dynamic range enlargement of slope-assisted BOTDA by using Brillouin phase-gain ratio," Journal of Lightwave Technology, vol. 35, no. 20, pp. 4451-4458, 2017.

[33] D. Zhou, Y. Dong, B. Wang et al., "Slope-assisted BOTDA based on vector sbs and frequency-agile technique for widestrain-range dynamic measurements," Optics Express, vol. 25, no. 3, pp. 1889-1902, 2017.

[34] D. Ba, B. Wang, D. Zhou et al., "Distributed measurement of dynamic strain based on multi-slope assisted fast BOTDA," Optics Express, vol. 24, no. 9, pp. 9781-9793, 2016.

[35] H. Zheng, D. Feng, J. Zhang et al., "Distributed vibration measurement based on a coherent multi-slope-assisted BOTDA with a large dynamic range," Optics Letters, vol. 44, no. 5, pp. 1245-1248, 2019.

[36] X. Tu, H. Luo, Q. Sun, X. Hu, and Z. Meng, "Performance analysis of slope-assisted dynamic BOTDA based on Brillouin gain or phase-shift in optical fibers," Journal of Optics, vol. 17, no. 10, pp. 105503-105503, 2015.

[37] A. Minardo, A. Coscetta, R. Bernini, and L. Zeni, "Heterodyne slope-assisted Brillouin optical time-domain analysis for dynamic strain measurements," Journal of Optics, vol. 18, no. 2, p. 025606, 2016.

[38] J. Hu, L. Xia, Y. Li, W. Quan, and X. Zhang, "Strain-induced vibration and temperature sensing BOTDA system combined frequency sweeping and slope-assisted techniques," Optics Express, vol. 24, no. 12, pp. 13610-13620, 2016.

[39] J. Marinelarena, J. Urricelqui, and A. Loayssa, "Enhancement of the dynamic range in slope-assisted coherent Brillouin optical time-domain analysis sensors," IEEE Photonics Journal, vol. 9, no. 3, pp. 1-10, 2017.

[40] G. Yang, X. Fan, B. Wang, and Z. He, "Enhancing strain dynamic range of slope-assisted BOTDA by manipulating Brillouin gain spectrum shape," Optics Express, vol. 26, no. 25, pp. 32 599-32 607, 2018.

[41] C. Feng, H. D. Bhatta, J. Bohbot et al., "Gain spectrum engineering in slope-assisted dynamic Brillouin optical timedomain analysis," Journal of Lightwave Technology, vol. 38, no. 24, pp. 6967-6975, 2020.

[42] D. Maraval, R. Gabet, Y. Jaouen, and V. Lamour, "Dynamic optical fiber sensing with Brillouin optical time domain reflectometry: application to pipeline vibration monitoring," Journal of Lightwave Technology, vol. 35, no. 16, pp. 3296-3302, 2017.

[43] A. Bergman, L. Yaron, T. Langer, and M. Tur, "Dynamic and distributed slope-assisted fiber strain sensing based on optical time-domain analysis of Brillouin dynamic gratings," Journal of Lightwave Technology, vol. 33, no. 12, pp. 2611-2616, 2015.

[44] A. Bergman, T. Langer, and M. Tur, "Coding-enhanced ultrafast and distributed Brillouin dynamic gratings sensing using coherent detection," Journal of Lightwave Technology, vol. 34, no. 24, pp. 5593-5600, 2016.

[45] B. Arik, L. Tomi, and T. Moshe, "Phase-based, high spatial resolution and distributed, static and dynamic strain sensing using brillouin dynamic gratings in optical fibers," Optics Express, vol. 25, no. 5, pp. 5376-5388, 2017.

[46] J. Morosi, M. Ferrarlo, P. Boffi, and M. Martinelli, "Double slope-assisted Brillouin optical correlation domain analysis," in 2017 Conference on Lasers and Electro-Optics Europe of European Quantum Electronics Conference (CLEO/EuropeEQEC), Munich, Germany, June 2017.

[47] Y. Wang, L. Zhao, M. Zhang et al., "Dynamic strain measurement by a single-slope-assisted chaotic Brillouin optical 
correlation-domain analysis," Optics Letters, vol. 45, no. 7, pp. 1822-1825, 2020.

[48] L. Zhao, Y. Wang, X. Hu et al., "Effect of chaotic time delay signature on Brillouin gain spectrum in the slope-assisted chaotic BOCDA," Optics Express, vol. 28, no. 12, 2020.

[49] H. Lee, N. Hayashi, Y. Mizuno, and K. Nakamura, "Slopeassisted Brillouin optical correlation-domain reflectometry: proof of concept," IEEE Photonics Journal, vol. 8, no. 3, pp. 1-7, 2016.

[50] H. Lee, N. Hayashi, Y. Mizuno, and K. Nakamura, "Operation of slope-assisted Brillouin optical correlation-domain reflectometry: comparison of system output with actual frequency shift distribution," Optics Express, vol. 24, no. 25, pp. 190197, 2016.

[51] H. Lee, N. Hayashi, Y. Mizuno, and K. Nakamura, "Slopeassisted Brillouin optical correlation-domain reflectometry using polymer optical fibers with high propagation loss," Journal of Lightwave Technology, vol. 35, no. 11, pp. 2306-2310, 2017.

[52] H. Lee, Y. Mizuno, and K. Nakamura, "Measurement sensitivity dependencies on incident power and spatial resolution in slopeassisted Brillouin optical correlation-domain reflectometry," Sensors and Actuators A: Physical, vol. 268, pp. 68-71, 2017.

[53] H. Lee, Y. Ochi, T. Matsui et al., "Distributed strain measurement and possible breakage detection of optical-fiberembedded composite structure using slope-assisted Brillouin optical correlation-domain reflectometry," Applied Physics Express, vol. 11, no. 7, article 072501, 2018.

[54] H. Lee, T. Ma, Y. Mizuno, and K. Nakamura, "Bending-lossindependent operation of slope-assisted Brillouin optical correlation-domain reflectometry," Scientific Reports, vol. 8, no. 1, article 7844, 2018.

[55] H. Lee, K. Noda, Y. Mizuno, and K. Nakamura, “Distributed temperature sensing based on slope-assisted Brillouin optical correlation-domain reflectometry with over $10 \mathrm{~km}$ measurement range," Electronics Letters, vol. 55, no. 5, pp. 276-278, 2019.

[56] H. Lee, K. Noda, Y. Mizuno, and K. Nakamura, “Trade-off relation between strain dynamic range and spatial resolution in slope-assisted Brillouin optical correlation-domain reflectometry," Measurement Science and Technology, vol. 30, no. 7, article 075204, 2019.

[57] H. Lee, Y. Mizuno, and K. Nakamura, "Enhanced stability and sensitivity of slope-assisted Brillouin optical correlationdomain reflectometry using polarization-maintaining fibers," OSA Continuum, vol. 2, no. 3, p. 874, 2019.

[58] H. Lee, K. Nakamura, and Y. Mizuno, "Recent progress in slope-assisted Brillouin optical correlation-domain reflectometry," Optical Fiber Technology, vol. 59, article 102312, 2020.

[59] W. Li, X. Bao, Y. Li, and L. Chen, "Differential pulse-width pair BOTDA for high spatial resolution sensing," Optics Express, vol. 16, no. 26, pp. 21616-21625, 2008.

[60] M. A. Soto, M. Taki, G. Bolognini, and F. D. Pasquale, "Optimization of a DPP-BOTDA sensor with $25 \mathrm{~cm}$ spatial resolution over $60 \mathrm{~km}$ standard single-mode fiber using simplex codes and optical pre-amplification," Optics Express, vol. 20, no. 7, pp. 6860-6869, 2012.

[61] S. M. Foaleng, M. Tur, J. Beugnot, and L. Thevenaz, "High spatial and spectral resolution long-range sensing using Brillouin echoes," Journal of Lightwave Technology, vol. 28, no. 20, pp. 2993-3003, 2010.
[62] A. W. Brown, B. G. Colpitts, and K. Brown, "Dark-pulse Brillouin optical time-domain sensor with 20 -mm spatial resolution," Journal of Lightwave Technology, vol. 25, no. 1, pp. 381-386, 2007.

[63] X. Zhang, J. Hu, and Y. Zhang, "A hybrid single-end-access BOTDA and COTDR sensing system using heterodyne detection," Journal of Lightwave Technology, vol. 31, no. 12, pp. 1954-1959, 2013.

[64] F. Wang, C. Zhu, C. Cao, and X. Zhang, "Enhancing the performance of BOTDR based on the combination of FFT technique and complementary coding," Optics Express, vol. 25, no. 4, pp. 3504-3513, 2017.

[65] N. Lalam, W. Ng, X. Dai, Q. Wu, and Y. Q. Fu, "Performance improvement of Brillouin ring laser based BOTDR system employing a wavelength diversity technique," Journal of Lightwave Technology, vol. 36, no. 4, pp. 1084-1090, 2018. 